\title{
Higher structures in algebraic quantum field theory
}

\author{
Marco Benini ${ }^{1}$ and Alexander Schenkel ${ }^{2, *}$
}

A brief overview of the recent developments of operadic and higher categorical techniques in algebraic quantum field theory is given. The relevance of such mathematical structures for the description of gauge theories is discussed.

\section{Background on AQFT}

Algebraic quantum field theory (AQFT) is a mathematical framework to formalize and investigate quantum field theories (QFTs) on Lorentzian manifolds, i.e. on spacetimes in the sense of general relativity. We have emphasized the adjective Lorentzian because this is what makes AQFT different from other mathematical approaches to QFT, such as (extended) topological QFT [1,54] or the factorization algebra approach of Costello and Gwilliam [26]. The original framework of Haag and Kastler [44] was restricted to QFTs on Minkowski spacetime, but a more flexible version of AQFT that works on all (globally hyperbolic) Lorentzian manifolds was later developed by Brunetti, Fredenhagen and Verch [23]. AQFT turns out to be a very powerful and successful framework not only for proving model-independent results for QFTs, but also for studying concrete applications with a high level of mathematical rigor. We refer to [21] for an overview of some of the recent advances in AQFT.

Before we can provide a definition of what an AQFT is, we have to make precise on which spacetimes we would like our QFTs to live. We refer to [3] for a concise introduction to Lorentzian geometry. In order to avoid pathologies, one typically considers only globally hyperbolic Lorentzian manifolds. These are Lorentzian manifolds $M$ for which there exists a Cauchy surface $\Sigma \subset M$, i.e. a codimension 1 hypersurface that is intersected precisely once by every inextensible causal curve. We further would like that $M$ is oriented and time-oriented in order to have a volume form and a way to distinguish between future and past. We collect all oriented and time-oriented globally hyperbolic Lorentzian manifolds (of a fixed dimension $m \geq 2$ ) in a category that we denote by Loc. The morphisms $f: M \rightarrow N$ in Loc are orientation and time- orientation preserving isometric embeddings of $M$ into $N$ such that the image $f(M) \subseteq N$ is open and causally convex, i.e. every causal curve in $N$ that starts and ends in $f(M)$ is entirely contained in $f(M)$. There exists a distinguished class $W \subseteq$ MorLoc of Loc-morphisms, called Cauchy morphisms, which is given by all $f: M \rightarrow N$ such that the image $f(M) \subseteq N$ contains a Cauchy surface of $N$. Loosely speaking, one should think of $f(M) \subseteq N$ as a "time slab" with respect to a time coordinate on $N$. The category Loc may be endowed with a further structure that encodes causal independence of subspacetimes. We call a pair of Loc-morphisms $\left(f_{1}: M_{1} \rightarrow N, f_{2}: M_{2} \rightarrow N\right)$ to a common target causally disjoint if their images $f_{1}\left(M_{1}\right) \subseteq N$ and $f_{2}\left(M_{2}\right) \subseteq N$ are causally disjoint subsets of $N$, i.e. there exists no causal curve connecting $f_{1}\left(M_{1}\right)$ and $f_{2}\left(M_{2}\right)$. The collection of all causally disjoint pairs of Loc-morphisms is denoted by $\perp_{\text {Loc }} \subseteq$ MorLoc $_{\mathrm{t}} \times{ }_{\mathrm{t}}$ MorLoc and we shall simply write $f_{1} \perp_{\text {Loc }} f_{2}$ whenever $\left(f_{1}, f_{2}\right) \in \perp_{\text {Loc }}$. The following variant of AQFT was proposed in [23] and it is called locally covariant QFT.

Definition 1.1. A locally covariant $Q F T$ is a functor $\mathfrak{A}$ : Loc $\rightarrow C^{*}$ Alg to the category of unital $C^{*}$-algebras that satisfies the following properties:

1. Isotony: For all Loc-morphisms $f: M \rightarrow N$, the $*-$ homomorphism $\mathfrak{A}(f): \mathfrak{A}(M) \rightarrow \mathfrak{A}(N)$ is injective.

2. Einstein causality: For all causally disjoint $f_{1} \perp_{\text {Loc }} f_{2}$, the induced commutator

$$
\left[\mathfrak{A}\left(f_{1}\right)(a), \mathfrak{A}\left(f_{2}\right)(b)\right]_{\mathfrak{A}(N)}=0
$$

is zero, for all $a \in \mathfrak{A}\left(M_{1}\right)$ and $b \in \mathfrak{A}\left(M_{2}\right)$.

3. Time-slice axiom: For all Cauchy morphisms $f \in W$, the *-homomorphism $\mathfrak{A}(f): \mathfrak{A}(M) \rightarrow \mathfrak{A}(N)$ is an isomorphism.

\footnotetext{
* Corresponding author

1 Fachbereich Mathematik, Universität Hamburg, Bundesstr. 55, 20146 Hamburg, Germany. E-mail: marco.benini@uni-hamburg.de

2 School of Mathematical Sciences, University of Nottingham, University Park, Nottingham NG7 2RD, United Kingdom.

E-mail: alexander.schenkel@nottingham.ac.uk
} 
The physical interpretation is as follows: The $C^{*}$ algebra $\mathfrak{A}(M) \in C^{*} \mathbf{A l g}$ associated to $M \in$ Loc describes the quantum observables of the theory that one can measure in the spacetime $M$. The $*$-homomorphism $\mathfrak{A}(f)$ : $\mathfrak{A}(M) \rightarrow \mathfrak{A}(N)$ associated to a Loc-morphism $f: M \rightarrow N$ pushes forward observables along this spacetime embedding. The isotony axiom then says that no observables are lost under such pushforwards, i.e. larger spacetimes are not allowed to have less observables than smaller ones. The Einstein causality axiom formalizes that spacelike separated observables commute with each other. Finally, the time-slice axiom implements a dynamical law or "time evolution" as it states that the observable algebra of a small region containing a Cauchy surface is isomorphic to the observable algebra of the full spacetime.

It might be a bit surprising for some readers that we did not mention Hilbert spaces in Definition 1.1, which are the predominant structures in other approaches to QFT. The reason is that AQFT splits the problem of describing a QFT in two separate steps: First, one constructs a theory in the sense of Definition 1.1, which describes the quantum observables as abstract $C^{*}$-algebras. Second, one studies (algebraic) states on these algebras, i.e. linear functionals $\omega_{M}: \mathfrak{A}(M) \rightarrow \mathbb{C}$ that are positive $\omega_{M}\left(a^{*} a\right) \geq 0$, for all $a \in \mathfrak{A}(M)$, and normalized $\omega_{M}(\mathbb{1})=1$. These states then define Hilbert spaces via the GNS-construction. This means that, while retaining a prominent role, in AQFT Hilbert spaces enter the game only at a later stage, when one analyzes the representation theory of a specific model. The advantage of axiomatizing only the observable algebras of a QFT, as it was done in Definition 1.1, is that one does not have to make any a priori choice of a distinguished "vacuum" state, but one treats all possible states on an equal footing. The reason why it is particularly important to do so is two-fold: 1.) different states can induce inequivalent Hilbert space representations, 2.) for QFTs on curved spacetimes there is no distinguished choice of vacuum state due to the lack of spacetime symmetries. Another more technical advantage is that the observable algebras of a QFT behave local (cf. Einstein causality in Definition 1.1), while states capture non-local quantum features such as entanglement. Hence, one can employ powerful local techniques for constructing and analyzing examples of AQFTs, which is particularly useful when discussing perturbatively interacting models and their renormalization, see e.g. [60] for a recent overview.

We would like to add some comments about variations of Definition 1.1 that are considered in the literature. We shall also explain why we think such variations are reasonable for certain purposes.
Variation 1.2. Instead of the category $C^{*}$ Alg of $C^{*}$ algebras, one may consider also other categories to describe the observables of a QFT. For example, one may consider the category *Alg of $*$-algebras or various categories of topological $*$-algebras, sometimes over the ring of formal power series $\mathbb{C}[[\hbar]]$. Such choices are useful for formalizing perturbatively interacting AQFTs, where one does not have $C^{*}$-norms.

Variation 1.3. Instead of the category Loc of all oriented and time-oriented globally hyperbolic spacetimes, one may consider also other categories of spacetimes. For example, one may take the full subcategory $\mathbf{L o c}_{\diamond} \subseteq$ Loc of all spacetimes $M$ whose underlying manifold is diffeomorphic to $\mathbb{R}^{m}$. Note that our notions of causal disjointness $\perp_{\text {Loc }}$ and Cauchy morphisms $W$ restrict to this subcategory, hence we can make sense of all axioms listed in Definition 1.1 for functors $\mathfrak{A}: \mathbf{L o c}_{\diamond} \rightarrow C^{*}$ Alg defined on this subcategory. Physically, one interprets such AQFTs as QFTs that are only defined on topologically trivial spacetimes. As another example, one may take the category COpen $(M)$ of causally convex open subsets $U \subseteq M$ of a fixed spacetime $M$. There exists an evident functor COpen $(M) \rightarrow$ Loc along which we can pull back our notion of causal disjointness $\perp_{\text {Loc }}$ and the Cauchy morphisms $W$. AQFTs on COpen $(M)$ describe QFTs that are defined on suitable subsets of a fixed spacetime $M$, which is in the spirit of the original Haag-Kastler approach [44].

Variation 1.4. The isotony axiom turns out to be too restrictive to capture important examples of QFTs that are sensitive to topological data. For example, the functor $\mathfrak{A}:$ Loc $\rightarrow C^{*}$ Alg describing gauge-invariant observables of Abelian Yang-Mills theory violates the isotony axiom because of electric and magnetic charges living in certain cohomology groups of the spacetimes. This feature was observed first in [28] and it was later refined and generalized in [4-7]. Our current practice is to drop the isotony axiom from the definition of AQFTs. In ongoing works, we attempt to find a suitable replacement by a descent (i.e. local-to-global) condition.

We would like to comment in more detail on the idea of introducing a descent condition for AQFT. Loosely speaking, descent means that the observable algebra $\mathfrak{A}(M)$ on a "complicated" spacetime $M$ can be obtained by patching together the observable algebras $\mathfrak{A}\left(U_{i}\right)$ on a suitable family of "simple" subspacetimes $U_{i} \subseteq M$. In practice, descent would allow us to replace questions about the complicated observable algebra $\mathfrak{A}(M)$ by a family of simpler questions about the algebras $\mathfrak{A}\left(U_{i}\right)$ and their interplay via embeddings. Such properties are prevalent in mathematics, with concrete manifestations given by sheaf or cosheaf conditions. To our surprise, it seems that descent in AQFT 
has not yet been studied systematically, at least according to our best knowledge. The reason for this might be that the most desirable kind of descent condition for AQFT, namely a cosheaf condition, turns out to be very restrictive and in particular it fails even in the simplest examples of non-interacting AQFTs. Recall that the cosheaf condition for the functor $\mathfrak{A}:$ Loc $\rightarrow C^{*}$ Alg underlying an AQFT states that, for every (suitable) cover $\left\{U_{i} \subseteq M\right\}$ by causally convex open subsets, the canonical morphism

$\operatorname{colim}\left(\coprod_{i j} \mathfrak{A}\left(U_{i j}\right) \rightrightarrows \coprod_{i} \mathfrak{A}\left(U_{i}\right)\right) \stackrel{\cong}{\rightrightarrows} \mathfrak{A}(M)$

is an isomorphism of $C^{*}$-algebras. Here $U_{i j}:=U_{i} \cap U_{j}$ denotes the intersections, $\amalg$ the coproduct in $C^{*} \mathbf{A l g}$ and colim the colimit in $C^{*}$ Alg. Looking at a simple example, we shall illustrate in Appendix A that it is very hard to find covers $\left\{U_{i} \subseteq M\right\}$ such that this condition holds true.

A weaker but still useful descent condition can be obtained by using ideas related to Fredenhagen's universal algebra [36-38]. We shall provide here only a rough sketch of the idea and refer to Section 2.3 for a precise implementation. Let us denote by $j: \mathbf{L o c}_{\diamond} \rightarrow$ Loc the full subcategory embedding from Variation 1.3. We may restrict any AQFT $\mathfrak{A}: \mathbf{L o c} \rightarrow C^{*}$ Alg to a functor $j^{*} \mathfrak{A}:=\mathfrak{A} \circ j: \mathbf{L o c}_{\diamond} \rightarrow C^{*} \mathbf{A l g}$ on $\mathbf{L o c}_{\diamond}$. It is easy to see that this functor satisfies the analogues for $\mathbf{L o c}_{\diamond}$ of the axioms in Definition 1.1, hence it is an AQFT that is however only defined on spacetimes with underlying manifold diffeomorphic to $\mathbb{R}^{m}$. Applying Fredenhagen's universal algebra construction to $j^{*} \mathfrak{A}$, i.e. forming the left Kan extension along the embedding functor $j:$ Loc $_{\diamond} \rightarrow$ Loc (cf. [53]), defines another functor $\operatorname{Lan}_{j} j^{*} \mathfrak{A}: \mathbf{L o c} \rightarrow C^{*} \mathbf{A l g}$ on all of Loc. Note that there exists a canonical comparison natural transformation $\epsilon_{\mathfrak{A}}: \operatorname{Lan}_{j} j^{*} \mathfrak{A} \rightarrow \mathfrak{A}$ given by the counit of the adjunction $\operatorname{Lan}_{j} \dashv j^{*}$. We hence may formalize a descent condition by demanding that $\epsilon_{\mathfrak{A}}$ is a natural isomorphism. In other words, this descent condition formalizes the idea that the AQFT $\mathfrak{A}$ on Loc is completely determined by its values on the category $\mathbf{L o c}_{\diamond} \subseteq$ Loc of spacetimes diffeomorphic to $\mathbb{R}^{m}$. This is similar to the descent condition in factorization homology [2], which is a topological variant of factorization algebras [26].

Let us mention the following issue with the latter descent condition, which will be addressed and solved in Section 2.3 once we have a more powerful mathematical machinery available. Notice that there is no reason why the functor $\operatorname{Lan}_{j} j^{*} \mathfrak{A}: \mathbf{L o c} \rightarrow C^{*}$ Alg should satisfy the axioms from Definition 1.1, i.e. $\operatorname{Lan}_{j} j^{*} \mathfrak{A}$ is not necessarily an AQFT in the sense of Definition 1.1, even if we drop the isotony axiom as in Variation 1.4. This has as a consequence that not even simple examples of AQFTs, such as the free Klein-Gordon theory, satisfy the present version of the descent condition. (They however satisfy a slightly weaker descent condition obtained by replacing Loc by the full subcategory $\mathbf{L o c}_{0} \subseteq$ Loc of connected spacetimes, cf. [53].) A solution to this problem is to use a more refined version of Fredenhagen's universal algebra construction that is obtained by methods from operad theory. This will be explained in Section 2.3.

\section{AQFT from an algebraic perspective}

The aim of this section is to identify a colored operad that controls the algebraic structures underlying AQFT. The main advantages of this operadic perspective are as follows: 1.) It provides a suitable framework for studying universal constructions for AQFTs, for example via operadic left Kan extensions. This will in particular allow us to formulate a precise version of the descent condition sketched in Section 1. 2.) It provides a suitable starting point for investigating higher structures in AQFT by importing ideas and techniques from the homotopy theory of algebras over operads, see e.g. $[45,46]$. The second point will be discussed in detail in Section 4. For details on the material presented below we refer to [13]. A generalization to other types of field theories (e.g. classical, linear, etc.) can be found in [20] and in Bruinsma's contribution to these proceedings [19].

\subsection{Orthogonal categories and AQFTs}

For the rest of this paper we shall adopt a very broad and flexible definition of AQFTs in which the spacetime category Loc is generalized to a so-called orthogonal category [13]. This allows us to treat different flavors of AQFTs, such as locally covariant QFTs, AQFTs on a fixed spacetime and chiral conformal AQFTs, on an equal footing.

Definition 2.1. An orthogonal category is a pair $\overline{\mathbf{C}}:=$ $(\mathbf{C}, \perp)$ consisting of a small category $\mathbf{C}$ and a subset $\perp \subseteq$ Mor $_{t} \times{ }_{t}$ MorC of the set of pairs of morphisms with a common target (called orthogonality relation), such that the following conditions hold true:

1. Symmetry: If $\left(f_{1}, f_{2}\right) \in \perp$, then $\left(f_{2}, f_{1}\right) \in \perp$.

2. o-Stability: If $\left(f_{1}, f_{2}\right) \in \perp$, then $\left(g f_{1} h_{1}, g f_{2} h_{2}\right) \in \perp$, for all composable C-morphisms $g, h_{1}$ and $h_{2}$.

We denote orthogonal pairs $\left(f_{1}, f_{2}\right) \in \perp$ also by $f_{1} \perp f_{2}$. An orthogonal functor $F: \overline{\mathbf{C}} \rightarrow \overline{\mathbf{D}}$ is a functor $F: \mathbf{C} \rightarrow \mathbf{D}$ such that $F\left(f_{1}\right) \perp_{\mathbf{D}} F\left(f_{2}\right)$ for all $f_{1} \perp_{\mathbf{C}} f_{2}$. We denote by OrthCat the category of orthogonal categories and orthogonal functors. 
Example 2.2. The pair $\overline{\mathbf{L O c}}=\left(\mathbf{L o c}, \perp_{\text {Loc }}\right)$ discussed in Section 1 is an orthogonal category. Endowing the subcategories $j: \operatorname{Loc}_{\diamond} \rightarrow$ Loc and $j_{M}: \operatorname{COpen}(M) \rightarrow$ Loc from Variation 1.3 with the pullback orthogonality relations $j^{*}\left(\perp_{\text {Loc }}\right)$ and $j_{M}^{*}\left(\perp_{\text {Loc }}\right)$ defines orthogonal categories $\overline{\operatorname{Loc}_{\diamond}}$ and $\overline{\operatorname{COpen}(M)}$. The embedding functors $j: \overline{\operatorname{Loc}_{\diamond}} \rightarrow \overline{\mathbf{L o c}}$ and $j_{M}: \overline{\mathbf{C O p e n}(M)} \rightarrow \overline{\mathbf{L o c}}$ are orthogonal functors. For an example that is not directly related to Lorentzian geometry, consider the category $\operatorname{Int}\left(\mathbb{S}^{1}\right)$ of open intervals $I \subset \mathbb{S}^{1}$ of the circle with morphisms given by subset inclusions $I \subseteq J \subset \mathbb{S}^{1}$. We define an orthogonality relation on $\operatorname{Int}\left(\mathbb{S}^{1}\right)$ by declaring two morphisms $I_{1}, I_{2} \subseteq J \subset \mathbb{S}^{1}$ to be orthogonal if and only if $I_{1} \cap I_{2}=\varnothing$ are disjoint intervals. The corresponding orthogonal category $\overline{\operatorname{Int}\left(\mathbb{S}^{1}\right)}$ features in chiral conformal QFT, see e.g. [52].

Throughout the whole section we shall fix a closed symmetric monoidal category $\mathbf{M}=(\mathbf{M}, \otimes, I)$, which we further assume to be bicomplete, i.e. all small limits and colimits exist in $\mathbf{M}$.

Definition 2.3. Let $\overline{\mathbf{C}}=(\mathbf{C}, \perp)$ be an orthogonal category. An M-valued AQFT on $\overline{\mathbf{C}}$ is a functor $\mathfrak{A}: \mathbf{C} \rightarrow \operatorname{Alg}_{\mathrm{As}_{\mathbf{s}}}(\mathbf{M})$ to the category of associative and unital algebras in $\mathbf{M}$ that satisfies the $\perp$-commutativity property: For all ( $f_{1}: c_{1} \rightarrow$ c) $\perp\left(f_{2}: c_{2} \rightarrow c\right)$, the diagram

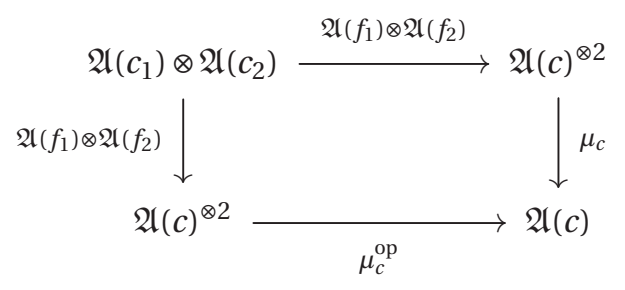

in $\mathbf{M}$ commutes, where $\mu_{c}^{(\mathrm{op})}$ denotes the (opposite) multiplication in the algebra $\mathfrak{A}(c)$. The category of $\mathbf{M}$-valued AQFTs on $\overline{\mathbf{C}}$ is defined as the full subcategory

$\mathbf{Q F T}(\overline{\mathbf{C}}) \subseteq \operatorname{Alg}_{\mathrm{As}}(\mathbf{M}){ }^{\mathrm{C}}$

of the functor category that consists of all $\perp$-commutative functors.

Remark 2.4. The following remarks are in order:

- Motivated by Variation 1.4, we decided to omit the isotony axiom in Definition 2.3 because it is often violated in examples.

- Comparing Definitions 2.3 and 1.1, it seems at first sight that we neglect the time-slice axiom in Definition 2.3. This is however not the case. We shall prove in Proposition 2.6 below that the time-slice axiom may be encoded by localizing the orthogonal category $\overline{\mathbf{L O c}}$ at all Cauchy morphisms $W \subseteq$ MorLoc, which defines another orthogonal category $\overline{\operatorname{Loc}\left[W^{-1}\right]}$.
- Note that Definition 2.3 does not refer explicitly to $*$ involutions on algebras. These can be included in a relatively straightforward way by choosing as target category $\mathbf{M}$ an involutive closed symmetric monoidal category, see [14] for the technical details.

Open Problem 2.5. Coming back to the last point of the previous remark, we would like to emphasize that, even though it is clear how to include $*$-algebras in Definition 2.3, capturing $C^{*}$-algebras as in Definition 1.1 is more subtle and still an open problem. The reason is that the category $C^{*}$ Alg of $C^{*}$-algebras is not (or at least not known to be) a category of $*$-algebras in a suitable involutive closed symmetric monoidal category. There are proposals in the literature $[33,34]$ to replace $C^{*} \mathbf{A l g}$ by the category of $*$-algebras in the involutive closed symmetric monoidal category of operator spaces in order to obtain a categorical approach to the theory of operator algebras. To the best of our knowledge, such an approach has not been applied to AQFT yet.

As promised in Remark 2.4, we shall now prove that Definition 2.3 includes the case of AQFTs satisfying the time-slice axiom. Let $\overline{\mathbf{C}}=(\mathbf{C}, \perp)$ be any orthogonal category and $W \subseteq$ MorC any subset of the set of morphisms. We denote by $\mathbf{C}\left[W^{-1}\right]$ the localization of the category $\mathbf{C}$ at $W$ and by $L: \mathbf{C} \rightarrow \mathbf{C}\left[W^{-1}\right]$ the corresponding localization functor. (We refer to [51, Section 7.1] for details on localizations of categories.) We define $\perp_{W}$ to be the smallest orthogonality relation on $\mathbf{C}\left[W^{-1}\right]$ such that $L\left(f_{1}\right) \perp_{W} L\left(f_{2}\right)$, for all $f_{1} \perp f_{2}$. This implies that $L: \overline{\mathbf{C}} \rightarrow \overline{\mathbf{C}\left[W^{-1}\right]}$ is an orthogonal functor. We shall denote by

$\mathbf{Q F T}(\overline{\mathbf{C}})^{W-\text { const }} \subseteq \mathbf{Q F T}(\overline{\mathbf{C}})$

the full subcategory of AQFTs satisfying the $W$-constancy property, i.e. $\mathfrak{A}(f): \mathfrak{A}(c) \rightarrow \mathfrak{A}\left(c^{\prime}\right)$ is an isomorphism in $\operatorname{Alg}_{\text {As }}(\mathbf{M})$, for all $\left(f: c \rightarrow c^{\prime}\right) \in W$.

Proposition 2.6. The pullback functor $L^{*}:=(-) \circ L$ : $\operatorname{Alg}_{\mathrm{As}}(\mathbf{M})^{\mathbf{C}\left[W^{-1}\right]} \rightarrow \operatorname{Alg}_{\mathrm{As}}(\mathbf{M})^{\mathbf{C}}$ on functor categories restricts to an equivalence of categories

$L^{*}: \mathbf{Q F T}\left(\overline{\mathbf{C}\left[W^{-1}\right]}\right) \stackrel{\sim}{\longrightarrow} \mathbf{Q F T}(\overline{\mathbf{C}})^{W-\text { const }}$.

Proof. One immediately observes that, for every $\mathfrak{B} \in$ $\mathbf{Q F T}\left(\overline{\mathbf{C}\left[W^{-1}\right]}\right) \subseteq \operatorname{Alg}_{\mathrm{As}}(\mathbf{M})^{\mathbf{C}\left[W^{-1}\right]}$, the functor $L^{*} \mathfrak{B}=\mathfrak{B} \circ L$ : $\mathbf{C} \rightarrow \operatorname{Alg}_{\mathrm{As}}(\mathbf{M})$ is $W$-constant and $\perp$-commutative. Hence, $L^{*}$ restricts to (2.4). By definition of localization, $L^{*}$ is fully faithful and hence so is its restriction (2.4) to full subcategories. It remains to show that (2.4) is essentially surjective. Given any $\mathfrak{A} \in \mathbf{Q F T}(\overline{\mathbf{C}})^{W-\text { const }}$, there exists by definition of localization a functor $\mathfrak{B}: \mathbf{C}\left[W^{-1}\right] \rightarrow \operatorname{Alg}_{\mathrm{As}}(\mathbf{M})$ and a natural isomorphism $\mathfrak{A} \cong L^{*} \mathfrak{B}$. One easily checks 
that $\mathfrak{B}$ is $\perp_{W}$-commutative, i.e. $\mathfrak{B} \in \mathbf{Q F T}\left(\overline{\mathbf{C}\left[W^{-1}\right]}\right)$, by using that the orthogonality relation $\perp_{W}$ is generated by $L\left(f_{1}\right) \perp_{W} L\left(f_{2}\right)$, for all $f_{1} \perp f_{2}$.

Example 2.7. Applying this general proposition to the orthogonal localization $L: \overline{\mathbf{L o c}} \rightarrow \overline{\operatorname{Loc}\left[W^{-1}\right]}$ of the usual spacetime category $\overline{\mathbf{L o c}}$ at all Cauchy morphisms $W$ shows that $\mathbf{Q F T}\left(\overline{\mathbf{L o c}\left[W^{-1}\right]}\right)$ is equivalent to the category of AQFTs satisfying also the time-slice axiom.

\subsection{AQFT operads}

From a category theoretical perspective, our Definition 2.3 of the category of AQFTs is neither elegant nor very effective because we select a certain full subcategory of a functor category by demanding additional properties. For example, it is a priori unclear if the category $\mathbf{Q F T}(\overline{\mathbf{C}})$ of AQFTs admits all limits and colimits. Another problem, which is related to our discussion of descent in Section 1, is as follows: Given an orthogonal functor $F: \overline{\mathbf{C}} \rightarrow \overline{\mathbf{D}}$, it is a priori unclear if its left Kan extension $\operatorname{Lan}_{F}: \operatorname{Alg}_{A s}(\mathbf{M})^{\mathbf{C}} \rightarrow \operatorname{Alg}_{A_{s}}(\mathbf{M})^{\mathbf{D}}$ restricts to a functor $\mathbf{Q F T}(\overline{\mathbf{C}}) \rightarrow \mathbf{Q F T}(\overline{\mathbf{D}})$ between the corresponding AQFT categories. More concretely, it is a priori unclear if constructions like Fredenhagen's universal algebra define $\perp$ commutative functors, i.e. bona fide AQFTs according to Definition 2.3. These problems were addressed and solved in [13], where we have shown that there exists a colored operad $\mathscr{O}_{\overline{\mathbf{C}}} \in \mathbf{O p}$ (Set) whose category of algebras is the AQFT category $\mathbf{Q F T}(\overline{\mathbf{C}})$. In this approach $\perp$ commutativity is not formulated as a property, instead it is encoded as a structure into the operad $\mathscr{O}_{\overline{\mathbf{C}}}$.

Let us recall that a colored operad $\mathscr{O} \in \mathbf{O p}$ (Set) with values in the category of sets Set is a generalization of the concept of a category where morphisms are allowed to have more than one input. The following picture visualizes this basic idea:

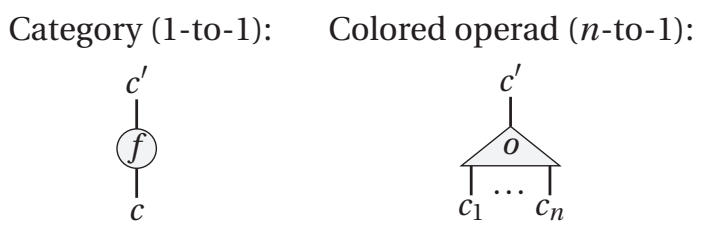

More precisely, a colored operad $\mathscr{O} \in \mathbf{O p}($ Set $)$ is described by the following data:

- an underlying set of objects, called colors in operad theory;

- for each tuple $(\underline{c}, t)=\left(\left(c_{1}, \ldots, c_{n}\right), t\right)$ of colors a set $\mathscr{O}\left(\begin{array}{c}t \\ c\end{array}\right) \in$ Set of operations from $\underline{c}$ to $t$;

- composition maps $\gamma: \overline{\mathscr{O}}\left(\begin{array}{l}t \\ \underline{c}\end{array}\right) \times \prod_{i=1}^{n} \mathscr{O}\left(\begin{array}{l}c_{i} \\ \underline{b}_{i}\end{array}\right) \rightarrow \mathscr{O}\left(\begin{array}{c}\left(\underline{b}_{1}, \ldots, \underline{b}_{n}\right) \\ )\end{array}\right)$;
- unit elements $\mathbb{1} \in \mathscr{O}\left(\begin{array}{l}t \\ t\end{array}\right)$;

- permutation group actions $\mathscr{O}(\sigma): \mathscr{O}\left(\begin{array}{c}t \\ c\end{array}\right) \rightarrow \mathscr{O}\left(\begin{array}{c}t \\ c \sigma\end{array}\right)$, where $\sigma \in \Sigma_{n}$ is a permutation of $n$ letters.

The composition maps are assumed to be associative in the obvious sense, unital with respect to the unit operations and also equivariant with respect to the permutation group actions. We refer to e.g. [65] for a detailed definition of colored operads.

The AQFT operad $\mathscr{O}_{\overline{\mathbf{C}}} \in \mathbf{O p}$ (Set) for an orthogonal category $\overline{\mathbf{C}}$ admits the following simple presentation by generators and relations [13]:

- Generators:

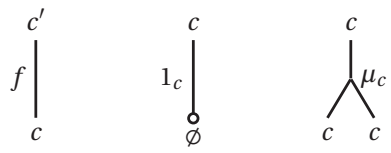

for all C-morphisms $f \in$ MorC and all objects $c \in \mathbf{C}$. The first generator describes the pushforward of observables along the spacetime embedding $f: c \rightarrow c^{\prime}$ and the other two generators the unit and multiplication of observables in the spacetime $c$.

- Functoriality relations:

$$
\left.\right|_{c} ^{c}=\left.\left.\operatorname{id}_{c}\right|_{c} ^{c} \quad f\right|_{c} ^{c^{\prime \prime}}=\left.f g\right|_{c} ^{c^{\prime \prime}}
$$

for all objects $c \in \mathbf{C}$ and all pairs of composable $\mathbf{C}$ morphisms $(f, g)$. The first relation means that the operadic units are identified with the identity morphisms of the category $\mathbf{C}$ and the second relation means that the operadic composition of $\mathbf{C}$-morphisms agrees with their categorical composition in $\mathbf{C}$.

- Algebra relations:
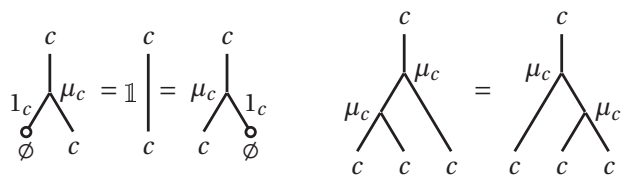

for all objects $c \in \mathbf{C}$. These relations express unitality and associativity of the multiplication of observables in the spacetime $c$.

- Compatibility relations:
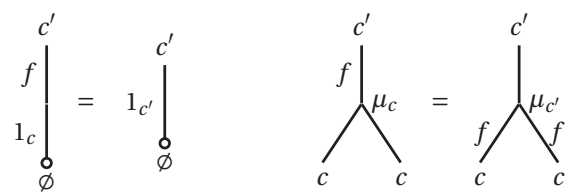
for all C-morphisms $f \in$ MorC. These relations express compatibility between the observable algebra structure on each spacetime and pushforwards of observables along spacetime embeddings.

$-\perp$-commutativity relations:

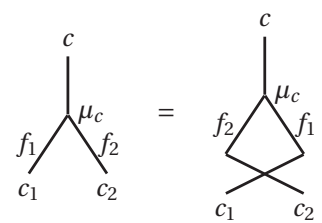

for all orthogonal pairs $f_{1} \perp f_{2}$ of $\mathbf{C}$-morphisms. These are the key relations that encode the $\perp$-commutativity property of Definition 2.3 as a structure into the colored operad $\mathscr{O}_{\overline{\mathbf{C}}}$.

As an alternative to this graphical description of the AQFT operad $\mathscr{O}_{\overline{\mathbf{C}}}$ by generators and relations, there is the following more algebraic description. See [13] for a proof of the following theorem.

Theorem 2.8. The AQFT operad $\mathscr{O}_{\overline{\mathbf{C}}} \in \mathbf{O p}$ (Set) described above is (isomorphic to) the colored operad specified by the following data:

- the set of colors is the set of objects of $\mathbf{C}$;

- the set of operations from $\underline{c}=\left(c_{1}, \ldots, c_{n}\right)$ to $t$ is the quotient set

$\mathscr{O}_{\overline{\mathbf{C}}}\left(\begin{array}{l}t \\ c\end{array}\right)=\left(\Sigma_{n} \times \prod_{i=1}^{n} \mathbf{C}\left(c_{i}, t\right)\right) / \sim_{\perp}$,

where $\Sigma_{n}$ is the permutation group on $n$ letters, $\mathbf{C}\left(c_{i}, t\right)$ are the Hom-sets of $\mathbf{C}$, and the equivalence relation is defined as follows: $(\sigma, \underline{f}) \sim_{\perp}\left(\sigma^{\prime}, \underline{f}^{\prime}\right)$ if and only if $f=$ $f^{\prime}$ and the right permutation $\sigma \sigma^{\prime-1}: f \sigma^{-1} \rightarrow f \sigma^{\prime-1}$ is generated by transpositions of adjacent orthogonal pairs;

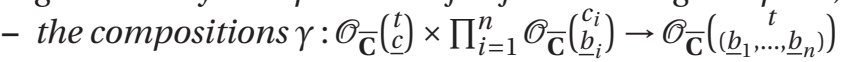
are

$$
\begin{aligned}
\gamma\left([\sigma, \underline{f}],\left(\left[\sigma_{1}, \underline{g}_{1}\right], \ldots,\left[\sigma_{n}, \underline{g}_{n}\right]\right)\right) \\
=\left[\sigma\left(\sigma_{1}, \ldots, \sigma_{n}\right), \underline{f}\left(\underline{g}_{1}, \ldots, \underline{g}_{n}\right)\right],
\end{aligned}
$$

where $\sigma\left(\sigma_{1}, \ldots, \sigma_{n}\right)=\sigma\left\langle k_{\sigma^{-1}(1)}, \ldots, k_{\sigma^{-1}(n)}\right\rangle\left(\sigma_{1} \oplus \cdots \oplus \sigma_{n}\right)$ is the product of the block permutation induced by $\sigma$ and the sum permutation induced by the $\sigma_{i}$, where $k_{i}$ is the length of the tuple $\underline{b}_{i}$, and $\underline{f}\left(\underline{g}_{1}, \ldots, \underline{g}_{n}\right)=$ $\left(f_{1} g_{11}, \ldots, f_{1} g_{1 k_{1}}, \ldots, f_{n} g_{n 1}, \ldots, f_{n} g_{n k_{n}}\right)$ is given by composition in the category $\mathbf{C}$;

- the units are $\left[e, \mathrm{id}_{t}\right] \in \mathscr{O}_{\overline{\mathbf{C}}}\left(\begin{array}{l}t \\ t\end{array}\right)$, where $e \in \Sigma_{1}$ is the identity permutation and $\operatorname{id}_{t}: t \rightarrow t$ the identity morphism in $\mathbf{C}$;

- the permutation actions are $\mathscr{O}_{\overline{\mathbf{C}}}\left(\sigma^{\prime}\right):[\sigma, \underline{f}] \mapsto\left[\sigma \sigma^{\prime}, \underline{f} \sigma^{\prime}\right]$.
The relevance of the AQFT operad $\mathscr{O}_{\overline{\mathbf{C}}} \in \mathbf{O p}$ (Set) is that its category of algebras is precisely the category $\mathbf{Q F T}(\overline{\mathbf{C}})$ of AQFTs defined in Definition 2.3. But what are algebras over operads? Loosely speaking, an $\mathbf{M}$-valued algebra over a colored operad $\mathscr{O} \in \mathbf{O p}($ Set $)$ is something like a "representation" of the operations described by $\mathscr{O}$ as $\mathbf{M}$ morphisms between a colored family of objects $A_{c} \in \mathbf{M}$, for all colors $c$. The following picture visualizes this basic idea:

$$
\overbrace{c_{1} \cdots T_{c_{n}}}^{c^{\prime}} \stackrel{\text { represent }}{\sim}\left(\bigotimes_{i=1}^{n} A_{c_{i}} \stackrel{A(o)}{\longrightarrow} A_{c^{\prime}}\right)
$$

Observe how the input and output colors match on both sides of this picture. There are of course certain compatibility conditions to be fulfilled, namely these $\mathbf{M}$ morphisms must be compatible with operadic compositions, operadic units and the permutation actions. We refer to e.g. [65] and [13] for a detailed definition of algebras over colored operads. The main theorem justifying the relevance of the colored operads $\mathscr{O}_{\overline{\mathbf{C}}} \in \mathbf{O p}$ (Set) is as follows. See [13] for a proof.

Theorem 2.9. For every orthogonal category $\overline{\mathbf{C}}$, the category $\operatorname{Alg}_{\mathscr{O}_{\overline{\mathbf{C}}}}(\mathbf{M})$ of algebras over the AQFT operad $\mathscr{O}_{\overline{\mathbf{C}}} \in$ $\mathbf{O p}$ (Set) is (isomorphic to) the category $\mathbf{Q F T}(\overline{\mathbf{C}})$ of $\mathbf{M}$ valued AQFTs on $\overline{\mathbf{C}}$ from Definition 2.3.

Remark 2.10. It is instructive to have a closer look at the action of the operations $[\sigma, f] \in \mathscr{O}_{\overline{\mathbf{C}}}(\underline{c})$ of the AQFT operad on a $\perp$-commutative functor $\mathfrak{A}: \mathbf{C} \rightarrow \operatorname{Alg}_{\text {As }}(\mathbf{M})$. One observes that the corresponding $\mathbf{M}$-morphism

$\mathfrak{A}([\sigma, \underline{f}]): \bigotimes_{i=1}^{n} \mathfrak{A}\left(c_{i}\right) \longrightarrow \mathfrak{A}(t)$

is concretely given by

$$
\begin{aligned}
& \mathfrak{A}([\sigma, \underline{f}])\left(a_{1} \otimes \cdots \otimes a_{n}\right) \\
& \quad=\mathfrak{A}\left(f_{\sigma^{-1}(1)}\right)\left(a_{\sigma^{-1}(1)}\right) \cdots \mathfrak{A}\left(f_{\sigma^{-1}(n)}\right)\left(a_{\sigma^{-1}(n)}\right) .
\end{aligned}
$$

In words, this means that one first pushes forward each observable $a_{i} \in \mathfrak{A}\left(c_{i}\right)$ along the spacetime embedding $f_{i}: c_{i} \rightarrow t$ and then multiplies the resulting observables in $\mathfrak{A}(t)$ according to the order prescribed by the permutation $\sigma$. Note that this is well-defined on the equivalence classes of operations (cf. Theorem 2.8) because $\mathfrak{A}$ is a $\perp$ commutative functor, i.e. one is allowed to interchange the order of multiplication for observables obtained by pushforward along orthogonal pairs of $\mathbf{C}$-morphisms. 


\subsection{Universal constructions}

The result of Theorem 2.9 that AQFTs are algebras over a colored operad $\mathscr{O}_{\overline{\mathbf{C}}} \in \mathbf{O p}($ Set $)$ is very useful for studying universal constructions in AQFT. For our first observation, let us recall that the target category $\mathbf{M}$ is by assumption bicomplete.

Proposition 2.11. For every orthogonal category $\overline{\mathbf{C}}$, the category $\mathbf{Q F T}(\overline{\mathbf{C}})$ of $\mathbf{M}$-valued AQFTs on $\overline{\mathbf{C}}$ admits all small limits and colimits.

Proof. By Theorem 2.9, we have that $\mathbf{Q F T}(\overline{\mathbf{C}}) \cong \operatorname{Alg}_{\mathscr{C}_{\overline{\mathbf{C}}}}(\mathbf{M})$ is the category of algebras over a colored operad. The latter category is known to be bicomplete whenever the target category $\mathbf{M}$ is bicomplete. See e.g. [42, Proposition 1.3.6] for a direct proof in the case of uncolored operads, which generalizes easily to colored operad.

Using this result, it is possible to build new AQFTs on $\overline{\mathbf{C}}$ by forming limits or colimits of diagrams of AQFTs on the same orthogonal category $\overline{\mathbf{C}}$. We expect that such constructions might be relevant for formalizing the algebraic adiabatic limit in perturbative AQFTs (see e.g. [22]), however we did not look into this in any detail.

Let us now consider a more interesting class of universal constructions that relate AQFTs on different orthogonal categories. Let us recall that such constructions are relevant e.g. for discussing descent, which involves AQFTs on both the spacetime category $\overline{\text { Loc }}$ and the category $\overline{\mathbf{L o c}_{\diamond}}$ of spacetimes whose underlying manifold is diffeomorphic to $\mathbb{R}^{m}$. The key observation that allows us to develop such constructions is that the assignment $\overline{\mathbf{C}} \mapsto \mathscr{O}_{\overline{\mathbf{C}}}$ of the AQFT operad to an orthogonal category is functorial $\mathscr{O}_{(-)}:$OrthCat $\rightarrow \mathbf{O p}($ Set). This means that given any orthogonal functor $F: \overline{\mathbf{C}} \rightarrow \overline{\mathbf{D}}$, we obtain a colored operad morphism $\mathscr{O}_{F}: \mathscr{O}_{\overline{\mathbf{C}}} \rightarrow \mathscr{O}_{\overline{\mathbf{D}}}$ and hence a pullback functor $\mathscr{O}_{F}^{*}: \operatorname{Alg}_{\mathscr{O}_{\overline{\mathbf{D}}}}(\mathbf{M}) \rightarrow \operatorname{Alg}_{\mathscr{O}_{\overline{\mathbf{C}}}}(\mathbf{M})$ between the corresponding categories of algebras. Under the natural identification $\mathbf{Q F T}(-) \cong \mathbf{A l g}_{\mathscr{O}_{(-)}}(\mathbf{M})$ given in Theorem 2.9, the pullback functor $\mathscr{O}_{F}^{*}$ gets identified with the restriction of the pullback functor $F^{*}:=(-) \circ F: \operatorname{Alg}_{\mathrm{As}}(\mathbf{M})^{\mathbf{D}} \rightarrow \operatorname{Alg}_{\mathrm{As}}(\mathbf{M}) \mathbf{C}$ to the full subcategories of AQFTs from Definition 2.3. This functor always admits a left adjoint.

Theorem 2.12. For every orthogonal functor $F: \overline{\mathbf{C}} \rightarrow \overline{\mathbf{D}}$, there exists an adjunction

$$
F_{!}: \mathbf{Q F T}(\overline{\mathbf{C}}) \rightleftarrows \mathbf{Q F T}(\overline{\mathbf{D}}): F^{*},
$$

where the right adjoint $F^{*}$ is the restriction of the pullback functor on functor categories to the subcategories of AQFTs.
Proof. The operadic pullback functor $\mathscr{O}_{F}^{*}: \operatorname{Alg}_{\mathscr{O}_{\overline{\mathbf{D}}}}(\mathbf{M}) \rightarrow$ $\mathbf{A l g}_{\mathscr{C}_{\overline{\mathbf{C}}}}(\mathbf{M})$ admits a left adjoint given by operadic left Kan extension, see e.g. [13] for a brief review. The natural identification of Theorem 2.9 then proves our claim.

Using the adjunctions from Theorem 2.12, one can develop and study interesting universal constructions that relate AQFTs on different orthogonal categories. Before looking at concrete examples inspired by physics, let us first note the following structural result for the full subcategory $\mathbf{Q F T}(\overline{\mathbf{C}}) \subseteq \operatorname{Alg}_{\mathrm{As}}(\mathbf{M})^{\mathbf{C}}$ of AQFTs from Definition 2.3. Given any orthogonal category $\overline{\mathbf{C}}=(\mathbf{C}, \perp)$, we form the orthogonal category $(\mathbf{C}, \varnothing)$ with the trivial (empty) orthogonality relation and observe that the identity functor defines an orthogonal functor $p_{\overline{\mathbf{C}}}:=\operatorname{id}_{\mathbf{C}}:(\mathbf{C}, \varnothing) \rightarrow \overline{\mathbf{C}}$. Note that $\mathbf{Q F T}(\mathbf{C}, \varnothing)=\operatorname{Alg}_{A_{s}}(\mathbf{M})^{\mathbf{C}}$ is the functor category. The following result is immediate.

Proposition 2.13. For every orthogonal category $\overline{\mathbf{C}}$, the adjunction

$$
p_{\overline{\mathbf{C}} !}: \operatorname{Alg}_{\mathrm{As}}(\mathbf{M}){ }^{\mathbf{C}} \rightleftarrows \mathbf{Q F T}(\overline{\mathbf{C}}): p_{\overline{\mathbf{C}}}^{*}
$$

corresponding to the canonical orthogonal functor $p_{\overline{\mathbf{C}}}=$ $\operatorname{id}_{\mathbf{C}}:(\mathbf{C}, \varnothing) \rightarrow \overline{\mathbf{C}}$ exhibits $\mathbf{Q F T}(\overline{\mathbf{C}})$ as a full reflective subcategory of the functor category $\operatorname{Alg}_{\mathrm{As}}(\mathbf{M}){ }^{\mathrm{C}}$.

A corollary of this result is that the left adjoint functor $F_{\text {! }}$ from Theorem 2.12 can be related to the ordinary left Kan extension $\operatorname{Lan}_{F}: \mathbf{A l g}_{A s}(\mathbf{M})^{\mathbf{C}} \rightarrow \operatorname{Alg}_{A s}(\mathbf{M})^{\mathbf{D}}$ of $\operatorname{Alg}_{\mathrm{As}}(\mathbf{M})$-valued functors.

Corollary 2.14. For every orthogonal functor $F: \overline{\mathbf{C}} \rightarrow \overline{\mathbf{D}}$, there exists a natural isomorphism of functors

$F_{!} \cong p_{\overline{\mathbf{D} !}} \circ \operatorname{Lan}_{F} \circ p_{\overline{\mathbf{C}}}{ }^{*}: \mathbf{Q F T}(\overline{\mathbf{C}}) \longrightarrow \mathbf{Q F T}(\overline{\mathbf{D}})$

where $F !$ is the left adjoint from Theorem 2.12, $\operatorname{Lan}_{F}$ the ordinary categorical left Kan extension and $p_{!} \dashv p^{*}$ the adjunction from Proposition 2.13.

Our first concrete example for an adjunction as in Theorem 2.12 is motivated physically by our goal to introduce a descent condition for AQFTs. Let $\overline{\mathbf{D}}$ be any orthogonal category and $\overline{\mathbf{C}} \subseteq \overline{\mathbf{D}}$ a full orthogonal subcategory, i.e. $\mathbf{C} \subseteq \mathbf{D}$ is a full subcategory such that $f_{1} \perp_{\mathbf{C}} f_{2}$ if and only if $f_{1} \perp_{\mathbf{D}} f_{2}$. For instance, $\overline{\mathbf{D}}$ may be the spacetime category $\overline{\mathbf{L O c}}$ from Example 2.2 and $\overline{\mathbf{C}}$ the category $\overline{\mathbf{L o c}_{\diamond}}$ of spacetimes whose underlying manifold is diffeomorphic to $\mathbb{R}^{m}$. In general, one should interpret $\overline{\mathbf{D}}$ as a category of "all spacetimes" and $\overline{\mathbf{C}} \subseteq \overline{\mathbf{D}}$ as a full subcategory of "nice spacetimes". Embedding the full subcategory defines an orthogonal functor that we shall denote by $j: \overline{\mathbf{C}} \rightarrow \overline{\mathbf{D}}$. As a 
consequence of Theorem 2.12, we obtain an adjunction

$$
j !: \mathbf{Q F T}(\overline{\mathbf{C}}) \rightleftarrows \mathbf{Q F T}(\overline{\mathbf{D}}): j^{*}
$$

between AQFTs on "nice spacetimes" and AQFTs on "all spacetimes". The right adjoint functor $j^{*}$ should be interpreted as a restriction functor that restricts an AQFT $\mathfrak{A} \in \mathbf{Q F T}(\overline{\mathbf{D}})$ that is defined on all of $\overline{\mathbf{D}}$ to an AQFT $j^{*} \mathfrak{A} \in$ $\mathbf{Q F T}(\overline{\mathbf{C}})$ on the full orthogonal subcategory $\overline{\mathbf{C}}$. More interestingly, the left adjoint $j_{\text {! }}$ is a universal extension functor that extends an AQFT $\mathfrak{B} \in \mathbf{Q F T}(\overline{\mathbf{C}})$ that is defined only on "nice spacetimes" in $\overline{\mathbf{C}} \subseteq \overline{\mathbf{D}}$ to all of $\overline{\mathbf{D}}$. In contrast to Fredenhagen's universal algebra construction [36-38,53], which is given by left Kan extension $\operatorname{Lan}_{j}: \operatorname{Alg}_{\mathrm{As}}(\mathbf{M})^{\mathbf{C}} \rightarrow$ $\operatorname{Alg}_{\mathrm{As}}(\mathbf{M})^{\mathbf{D}}$ of the underlying functors, our left adjoint $j$ ! always defines an AQFT j!: $\mathfrak{B} \in \mathbf{Q F T}(\overline{\mathbf{D}})$ on $\overline{\mathbf{D}}$ and not only a functor $\operatorname{Lan}_{j} \mathfrak{B}: \mathbf{D} \rightarrow \operatorname{Alg}_{\mathrm{As}}(\mathbf{M})$ that might violate the $\perp$-commutativity axiom from Definition 2.3. (It was shown in [13] that $\operatorname{Lan}_{j} \mathfrak{B}$ violates $\perp$-commutativity on non-connected spacetimes.) The following result states an important technical property of the adjunction (2.18).

Proposition 2.15. For every full orthogonal subcategory embedding $j: \overline{\mathbf{C}} \rightarrow \overline{\mathbf{D}}$, the adjunction (2.18) exhibits $\mathbf{Q F T}(\overline{\mathbf{C}})$ as a full coreflective subcategory of $\mathbf{Q F T}(\overline{\mathbf{D}})$.

Remark 2.16. Let us explain in more detail why this result is crucial for the interpretation of $j^{*}$ as a restriction functor and $j$ ! as an extension functor. Given an AQFT $\mathfrak{B} \in \mathbf{Q F T}(\overline{\mathbf{C}})$, we form the extension $j ! \mathfrak{B} \in \mathbf{Q F T}(\overline{\mathbf{D}})$ and ask if this alters the values of the AQFT on the subcategory $\overline{\mathbf{C}} \subseteq \overline{\mathbf{D}}$, i.e. if the restriction $j^{*} j ! \mathfrak{B}$ of the extension is isomorphic to the original theory $\mathfrak{B}$. Proposition 2.15 states that this is the case and that the unit $\eta_{\mathfrak{B}}: \mathfrak{B} \rightarrow j^{*} j ! \mathfrak{B}$ of the adjunction (2.18) provides such an isomorphism. In other words, the extension functor $j$ ! does not alter the values of AQFTs on the full subcategory $\overline{\mathbf{C}} \subseteq \overline{\mathbf{D}}$.

We now can formalize our sketchy ideas from Section 1 about a descent condition in AQFT.

Definition 2.17. An AQFT $\mathfrak{A} \in \mathbf{Q F T}(\overline{\mathbf{D}})$ is called $j$-local if the corresponding component $\epsilon_{\mathfrak{A}}: j_{!} j^{*} \mathfrak{A} \rightarrow \mathfrak{A}$ of the counit of the adjunction (2.18) is an isomorphism. We denote the full subcategory of $j$-local AQFTs by $\mathbf{Q F T}(\overline{\mathbf{D}})^{j-\mathrm{loc}} \subseteq \mathbf{Q F T}(\overline{\mathbf{D}})$.

Corollary 2.18. For every full orthogonal subcategory embedding $j: \overline{\mathbf{C}} \rightarrow \overline{\mathbf{D}}$, the adjunction (2.18) restricts to an adjoint equivalence $j_{!}: \mathbf{Q F T}(\overline{\mathbf{C}}) \rightleftarrows \mathbf{Q F T}(\overline{\mathbf{D}})^{j-\text { loc }}: j^{*}$.

Remark 2.19. The physical interpretation is that $j$-local AQFTs $\mathfrak{A} \in \mathbf{Q F T}(\overline{\mathbf{D}})^{j-\text { loc }}$ are those AQFTs on $\overline{\mathbf{D}}$ that are completely determined by their restriction to the subcategory $\overline{\mathbf{C}} \subseteq \overline{\mathbf{D}}$. In the case of $\overline{\mathbf{L o c}_{\diamond}} \subseteq \overline{\mathbf{L o c}}$, this means that the value $\mathfrak{A}(M)$ of a $j$-local AQFT on any spacetime $M \in$ Loc is completely determined by the values of $\mathfrak{A}$ on the sub-

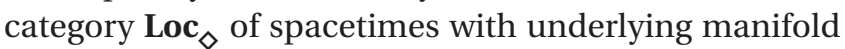
diffeomorphic to $\mathbb{R}^{m}$. Hence, $j$-locality is a type of descent condition for AQFTs.

Example 2.20. From the results in [53] and [13] one can conclude that the free Klein-Gordon AQFT is $j$-local in the above sense for $j: \overline{\mathbf{L o c}_{\diamond}} \rightarrow \overline{\mathbf{L o c}}$.

Our second concrete example for an adjunction as in Theorem 2.12 is motivated by the time-slice axiom of AQFTs. Let $\overline{\mathbf{C}}$ be any orthogonal category and $W \subseteq$ MorC any subset of the set of morphisms. The corresponding orthogonal localization functor $L: \overline{\mathbf{C}} \rightarrow \overline{\mathbf{C}\left[W^{-1}\right]}$ (see the text before Proposition 2.6) defines an adjunction

$$
L_{!}: \mathbf{Q F T}(\overline{\mathbf{C}}) \rightleftarrows \mathbf{Q F T}\left(\overline{\mathbf{C}\left[W^{-1}\right]}\right): L^{*}
$$

between AQFTs on $\overline{\mathbf{C}}$ and AQFTs on $\overline{\mathbf{C}\left[W^{-1}\right]}$. The following result is a direct consequence of Proposition 2.6.

Proposition 2.21. For every orthogonal localization $L$ : $\overline{\mathbf{C}} \rightarrow \overline{\mathbf{C}\left[W^{-1}\right]}$, the right adjoint functor $L^{*}$ in (2.19) is fully faithful and its essential image is the full subcategory $\mathbf{Q F T}(\overline{\mathbf{C}})^{W-\text { const }} \subseteq \mathbf{Q F T}(\overline{\mathbf{C}})$ of $W$-constant AQFTs. Hence, the adjunction (2.19) exhibits $\mathbf{Q F T}\left(\overline{\mathbf{C}\left[W^{-1}\right]}\right)$ as a full reflective subcategory of $\mathbf{Q F T}(\overline{\mathbf{C}})$ and it restricts to an adjoint equivalence $L_{!}: \mathbf{Q F T}(\overline{\mathbf{C}})^{W \text {-const }} \rightleftarrows \mathbf{Q F T}\left(\overline{\mathbf{C}\left[W^{-1}\right]}\right): L^{*}$.

An immediate corollary of this result is that there exist equivalent characterizations of $W$-constant AQFTs.

Corollary 2.22. Let $\mathfrak{A} \in \mathbf{Q F T}(\overline{\mathbf{C}})$. Then the following are equivalent:

1. $\mathfrak{A}$ is $W$-constant, i.e. for all $f \in W$ the $\mathbf{A l g}_{\mathrm{As}}(\mathbf{M})$ morphism $\mathfrak{A}(f): \mathfrak{A}(c) \rightarrow \mathfrak{A}\left(c^{\prime}\right)$ is an isomorphism.

2. The component $\eta_{\mathfrak{A}}: \mathfrak{A} \rightarrow L^{*} L ! \mathfrak{A}$ of the unit of the adjunction (2.19) is an isomorphism.

Remark 2.23. We would like to stress that our adjunctions from this section are not only theoretically interesting, but they already found concrete applications to physical problems. We refer to [8] for a study of AQFTs on spacetimes with time-like boundaries from this perspective.

\section{Higher structures in gauge theory}

The aim of this section is to explain in rather nontechnical terms the structural differences between "ordinary" field theories, such as Klein-Gordon theory, and gauge theories, such as Yang-Mills theory. The latter are 
instances of higher structures and therefore require refined concepts of category theory for their formalization, e.g. $\infty$-category theory $[55,56]$ or model category theory $[32,50]$. The incorporation of such higher structures into AQFT will be discussed in Section 4 .

\subsection{Groupoids of gauge fields}

The main difference between "ordinary" field theories and gauge theories is of course the presence of gauge symmetries. Even though this observation sounds like a tautology, it has profound consequences on how one should think of the spaces of fields in these situations. For "ordinary" theories, the collection of all fields $\mathfrak{F}$ has the structure of a set, i.e. given two fields $\Phi, \Phi^{\prime} \in \mathfrak{F}$, we can ask if these fields are the same or not by testing whether $\Phi=\Phi^{\prime}$ holds true. In a gauge theory this becomes more complicated because in addition to gauge fields $A$ one also has gauge transformations $A \stackrel{g}{\longrightarrow} A^{\prime}$ between gauge fields. Hence, the collection of all gauge fields $\mathfrak{G}$ has the structure of a groupoid and not that of a set! The following picture visualizes the basic idea:
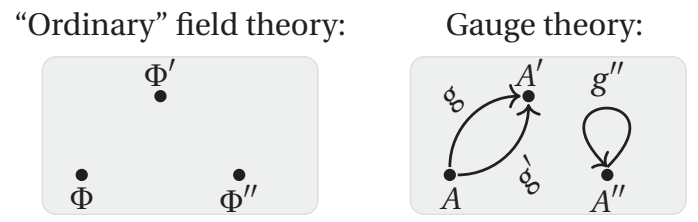

This groupoid structure drastically changes the way one should think of two gauge fields as being the same. In contrast to sets, being the same in a groupoid is not anymore a property, but rather a structure in the sense that one needs a gauge transformation $A \stackrel{g}{\longrightarrow} A^{\prime}$ in order to witness that $A$ and $A^{\prime}$ are the same. As visualized in (3.1), there might exist different witnesses for two gauge fields being the same, and in particular there are generically non-trivial "loops" in the groupoid of gauge fields. These loops should be understood as higher order structures in the groupoid of gauge fields that cannot be seen at the level of the naive "gauge orbit space". Recall that the naive "gauge orbit space" is obtained by forming gauge equivalence classes of gauge fields, i.e. it is the zeroth homotopy group $\pi_{0} \mathfrak{G}$ of the groupoid of gauge fields $\mathfrak{G}$. This construction however neglects information on the loops in $\mathfrak{G}$, which is contained in the first homotopy groups $\pi_{1}(\mathfrak{G}, A)$, for $A \in \mathfrak{G}$. Hence, the groupoid of gauge fields $\mathfrak{G}$ includes more refined information on the gauge theory than the naive "gauge orbit space". We shall explain later why this additional information is crucial.
Remark 3.1. The same way of reasoning of course also applies to gauge transformations themselves. In particular, if there are gauge transformations of gauge transformations, then the collection of gauge fields is described by a 2-groupoid. If there are gauge transformations of gauge transformations of gauge transformations, then one gets a 3-groupoid, and so on. Hence, the natural framework in which to study gauge theories and higher gauge theories is that of $\infty$-groupoids. Because of the chain of inclusions

Set $\hookrightarrow \operatorname{Grpd} \hookrightarrow 2 \operatorname{Grpd} \hookrightarrow \cdots \hookrightarrow \infty$ Grpd

all ordinary field theories, gauge theories, 2-gauge theories, ..., can be regarded as particular examples of theories in the sense of $\infty$-groupoids. In what follows we will mostly focus on the case of 1-groupoids in order to simplify our presentation. However, everything said below generalizes to $\infty$-groupoids and in particular our model categorical framework for AQFT in Section 4 applies to higher gauge theories as well.

Example 3.2. As a very concrete example, let us consider principal $G$-bundles with connections on a Cartesian space $U \cong \mathbb{R}^{m}$. Because all principal $G$-bundles on $U$ are trivializable, the groupoid of gauge fields on $U$ is

$B G^{\operatorname{con}}(U)=\left\{\begin{array}{ll}\text { Obj: } & A \in \Omega^{1}(U, \mathfrak{g}) \\ \text { Mor: } & A \stackrel{g}{\longrightarrow} A \triangleleft g:=g^{-1} A g+g^{-1} \mathrm{~d} g \\ & \text { with } g \in C^{\infty}(U, G)\end{array}\right.$,

where $\mathfrak{g}$ is the Lie algebra of the structure Lie group $G$. For the Abelian cases $G=U(1)$ or $G=\mathbb{R}$, one easily computes the homotopy groups and obtains $\pi_{0} B G^{\mathrm{con}}(U) \cong$ $\Omega^{1}(U) / \mathrm{d} \Omega^{0}(U)$ and $\pi_{1}\left(B G^{\mathrm{con}}(U), A\right) \cong G$. Hence, the naive "gauge orbit space" does not distinguish between the two different structure groups, but the higher order information contained in $\pi_{1}$ does. In other words, the groupoid perspective on gauge theory is truly more refined than the naive "gauge orbit space" perspective.

Working with groupoids requires some additional care because the correct notion of two groupoids being the same is via categorical equivalence rather than isomorphism. This is because the category of groupoids Grpd is actually a 2-category, whose objects are all groupoids $\mathfrak{G}$, 1-morphisms are functors $F: \mathfrak{G} \rightarrow \mathfrak{G}^{\prime}$ and 2-morphisms are natural isomorphisms $\zeta: F \rightarrow F^{\prime}$ between functors $F, F^{\prime}: \mathfrak{G} \rightarrow \mathfrak{G}^{\prime}$. The equivalences in this 2-category are the usual categorical equivalences, i.e. functors $F: \mathfrak{G} \rightarrow \mathfrak{G}^{\prime}$ that can be "inverted up to 2-morphisms" in the sense that there exists a functor $F^{\prime}: \mathfrak{G}^{\prime} \rightarrow \mathfrak{G}$, going in the opposite direction, together with natural isomorphisms $F^{\prime} F \cong \mathrm{id}_{\mathfrak{G}}$ 


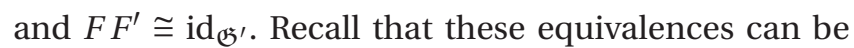
characterized as fully faithful and essentially surjective functors $F: \mathfrak{G} \rightarrow \mathfrak{G}^{\prime}$. As a side-remark, let us briefly mention that the category of $\infty$-groupoids is not only a 2category, but actually an $\infty$-category [55]. Hence, the higher the gauge theory one considers, the higher one has to climb up on the categorical ladder.

For our purposes, it will be convenient to adopt a slightly different, but related, point of view and regard Grpd (and also $\infty$ Grpd) as a model category. A model category is a bicomplete category $\mathbf{C}$ that is endowed with three distinguished classes of morphisms - called weak equivalences, fibrations and cofibrations - that have to satisfy a list of conditions. See e.g. [32,50] for details. These axioms are designed in such a way that the weak equivalences define a consistent notion of two objects being the same. In particular, this notion is preserved under certain (derived) functorial constructions. Let us expand on the latter point because it is crucial. Given any functor $F: \mathbf{C} \rightarrow \mathbf{D}$ between two model categories $\mathbf{C}$ and $\mathbf{D}$, it is in general not true that $F$ maps weak equivalences in $\mathbf{C}$ to weak equivalences in $\mathbf{D}$. This will of course introduce inconsistencies, because weakly equivalent objects are regarded as being the same according to the philosophy of model category theory. Considering only those functors $F: \mathbf{C} \rightarrow \mathbf{D}$ that do preserve weak equivalences would be too restrictive, because several natural constructions, e.g. limit and colimit functors, are not of this type. The way out of this dilemma is to "deform" (in a controlled way) the functor $F: \mathbf{C} \rightarrow \mathbf{D}$ to obtain a functor that does preserve weak equivalences. That is precisely what derived functors do for us! The usual context in which the theory of derived functors applies is when one has a Quillen adjunction between model categories, i.e. an adjunction $F: \mathbf{C} \rightleftarrows \mathbf{D}: G$ in which the right adjoint functor $G$ preserves fibrations and acyclic fibrations (i.e. morphisms that are both a fibration and a weak equivalence). Choosing a natural cofibrant replacement $\left(Q: \mathbf{C} \rightarrow \mathbf{C}, q: Q \stackrel{\sim}{\rightarrow} \mathrm{id}_{\mathbf{C}}\right)$ for $\mathbf{C}$ and a natural fibrant replacement $\left(R: \mathbf{D} \rightarrow \mathbf{D}, r: \operatorname{id}_{\mathbf{D}} \stackrel{\sim}{\rightarrow} R\right)$ for $\mathbf{D}$, one can define the left derived functor

$\mathbb{L} F:=F Q: \mathbf{C} \longrightarrow \mathbf{D}$

and the right derived functor

$\mathbb{R} G:=G R: \mathbf{D} \longrightarrow \mathbf{C}$

corresponding to the Quillen adjunction $F \dashv G$. It can be shown that both derived functors preserve weak equivalences and that different choices of (co)fibrant replacements define naturally weakly equivalent derived functors $[32,50]$.

Let us now look at model categories and derived functors in action in order to better understand what they do for us and why they are crucial. We first recall that the category Grpd of groupoids is a model category with respect to the following choices (see e.g. [48]): A morphism $F: \mathfrak{G} \rightarrow \mathfrak{H}$ (i.e. functor) between two groupoids is - a weak equivalence if it is fully faithful and essentially surjective;

- a fibration if it is an isofibration, i.e. for each object $x \in \mathfrak{G}$ and $\mathfrak{H}$-morphism $g: F(x) \rightarrow y$ there exists a $\mathfrak{G}$ morphism $f: x \rightarrow x^{\prime}$ such that $F(f)=g$;

- a cofibration if it is injective on objects.

Note that the weak equivalences in this model structure are precisely the equivalences one obtains when thinking of Grpd as a 2-category. Given any small category $\mathbf{D}$, we consider the functor category $\mathbf{G r p d}^{\mathbf{D}}$ of all functors from D to Grpd, which we interpret as diagrams of shape D in Grpd. The constant diagram functor const : Grpd $\rightarrow$ Grpd $^{\mathbf{D}}$ admits both a left and a right adjoint functor, respectively given by the colimit functor colim : $\mathbf{G r p d}^{\mathbf{D}} \rightarrow$ Grpd and the limit functor lim : $\mathbf{G r p d}^{\mathbf{D}} \rightarrow$ Grpd. Let us focus on the adjunction const $\dashv$ lim, whose right adjoint is the limit functor, and note that this is a Quillen adjunction when one endows Grpd $^{\mathbf{D}}$ with the injective model category structure, i.e. a $\mathbf{G r p d}^{\mathbf{D}}$-morphism $\zeta: X \rightarrow Y$ is a cofibration (respectively, a weak equivalence) if all components $\zeta_{d}: X(d) \rightarrow Y(d)$, for $d \in \mathbf{D}$, are cofibrations (respectively, weak equivalences) in Grpd. The corresponding right derived functor

holim := $\mathbb{R} \lim : \operatorname{Grpd}^{\mathbf{D}} \longrightarrow$ Grpd

is called the homotopy limit functor. In contrast to the ordinary limit functor lim, the homotopy limit functor holim has the important property that it preserves weak equivalences.

For our gauge-theoretic example below, we shall need a concrete model for homotopy limits of cosimplicial groupoids, see e.g. [48] for details. Let $\mathbf{D}=\Delta$ be the simplex category and consider the corresponding functor category $\mathbf{G r p d}^{\Delta}$. An object $\mathfrak{G}^{\bullet} \in \mathbf{G r p d}^{\Delta}$ is a cosimplicial groupoid, which one may visualize as follows

$\mathfrak{G}^{\bullet}=\left(\mathfrak{G}^{0} \underset{d^{1}}{\stackrel{d^{0}}{\rightrightarrows}} \mathfrak{G}^{1} \rightrightarrows \mathfrak{G}^{2} \underset{\rightrightarrows}{\rightrightarrows} \cdots\right)$

where as usual we suppressed the codegeneracy maps $s^{i}$.

Lemma 3.3. Let $\mathfrak{G}^{\bullet} \in \mathbf{G r p d}^{\Delta}$ be any cosimplicial groupoid. The following groupoid defines a model for the homotopy limit holim $\mathfrak{G}^{\bullet} \in$ Grpd:

- objects are pairs $(x, h)$ consisting of an object $x \in \mathfrak{G}^{0}$ and a $\mathfrak{G}^{1}$-morphism $h: d^{1}(x) \rightarrow d^{0}(x)$, such that $s^{0}(h)=\mathrm{id}_{x}$ and $d^{0}(h) \circ d^{2}(h)=d^{1}(h)$ in $\mathfrak{G}^{2}$; 
- morphisms $g:(x, h) \rightarrow\left(x^{\prime}, h^{\prime}\right)$ are $\mathfrak{G}^{0}$-morphisms $g:$ $x \rightarrow x^{\prime}$, such that the diagram

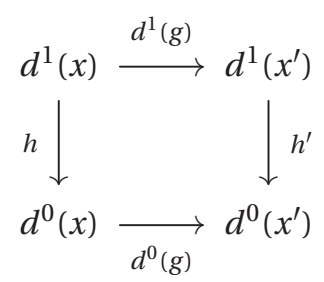

in $\mathfrak{G}^{1}$ commutes.

Remark 3.4. We note that in general the homotopy limit holim $\mathfrak{G}^{\bullet}$ is not weakly equivalent to the ordinary limit $\lim \mathfrak{G}^{\circ}$. The latter is given by the groupoid whose objects are all $x \in \mathfrak{G}^{0}$ satisfying the equality $d^{1}(x)=d^{0}(x)$ and whose morphisms are all $\mathfrak{G}^{0}$-morphisms $g: x \rightarrow x^{\prime}$ between such objects that additionally satisfy $d^{1}(g)=d^{0}(g)$. The homotopy limit is weaker than the ordinary limit in the sense that the equality $d^{1}(x)=d^{0}(x)$ is promoted to the additional datum of a $\mathfrak{G}^{1}$-morphism $h: d^{1}(x) \rightarrow d^{0}(x)$ witnessing that $d^{1}(x)$ and $d^{0}(x)$ are isomorphic objects in $\mathfrak{G}^{1}$. This will be crucial in the example below.

Example 3.5. Recall from Example 3.2 the groupoid $B G^{\mathrm{con}}(U) \in$ Grpd of gauge fields with structure group $G$ on a Cartesian space $U$. The assignment $U \mapsto B G^{\text {con }}(U)$ is contravariantly functorial on the category Cart of Cartesian spaces, i.e. we have a functor $B G^{\text {con }}:$ Cart $^{\text {op }} \rightarrow$ Grpd. We now shall show that homotopy limits allow us to compute from this information the groupoid of gauge fields on a general manifold $M$. Let us choose any good open cover $\left\{U_{i} \subseteq M\right\}$ and form its Čech nerve

$U_{\bullet}:=\left(\coprod_{i} U_{i} \leftleftarrows \coprod_{i j} U_{i j} \leftleftarrows \cdots\right)$,

where as usual we denote intersections by $U_{i_{1} \ldots i_{n}}:=U_{i_{1}} \cap$ $\cdots \cap U_{i_{n}}$. Using that by hypothesis all non-empty intersections are Cartesian spaces, we can apply the functor $B G^{\mathrm{con}}:$ Cart $^{\mathrm{op}} \rightarrow$ Grpd and obtain a cosimplicial groupoid

$B G^{\mathrm{con}}\left(U_{\bullet}\right):=\left(\prod_{i} B G^{\mathrm{con}}\left(U_{i}\right) \rightrightarrows \prod_{i j} B G^{\mathrm{con}}\left(U_{i j}\right) \rightrightarrows \cdots\right)$

associated to the cover $\left\{U_{i} \subseteq M\right\}$. Computing the corresponding homotopy $\operatorname{limit} \operatorname{holim} B G^{\mathrm{con}}\left(U_{\bullet}\right) \in$ Grpd according to Lemma 3.3, we obtain the groupoid whose

- objects are pairs of families $\left(\left\{A_{i} \in \Omega^{1}\left(U_{i}, \mathfrak{g}\right)\right\},\left\{g_{i j} \in\right.\right.$ $\left.\left.C^{\infty}\left(U_{i j}, G\right)\right\}\right)$, satisfying

- $\left.A_{j}\right|_{U_{i j}}=\left.A_{i}\right|_{U_{i j}} \triangleleft g_{i j}$, for all $i, j$,

- $g_{i i}=e$ is the identity of $G$, for all $i$, and
- the cocycle condition $\left.\left.g_{i j}\right|_{U_{i j k}} g_{j k}\right|_{U_{i j k}}=\left.g_{i k}\right|_{U_{i j k}}$, for all $i, j, k$;

- morphisms $\left(\left\{A_{i}\right\},\left\{g_{i j}\right\}\right) \rightarrow\left(\left\{A_{i}^{\prime}\right\},\left\{g_{i j}^{\prime}\right\}\right)$ are families $\left\{h_{i} \in\right.$ $\left.C^{\infty}\left(U_{i}, G\right)\right\}$, satisfying

- $A_{i}^{\prime}=A_{i} \triangleleft h_{i}$, for all $i$, and

- $g_{i j}^{\prime}=\left.\left.h_{i}^{-1}\right|_{U_{i j}} g_{i j} h_{j}\right|_{U_{i j}}$, for all $i, j$.

Observe that this groupoid is precisely the groupoid of gauge fields on $M$, expressed in terms of Čech data with respect to the good open cover $\left\{U_{i} \subseteq M\right\}$. In contrast to this, the ordinary $\operatorname{limit} \lim B G^{\mathrm{con}}\left(U_{\bullet}\right) \in \mathbf{G r p d}$ is given by the groupoid whose objects are 1-forms $A \in$ $\Omega^{1}(M, \mathfrak{g})$ on $M$ and whose morphisms are $A \rightarrow A \triangleleft h$, with $h \in C^{\infty}(M, G)$. Note that the latter groupoid describes only gauge fields on the trivial principal $G$-bundle $\operatorname{pr}_{1}: M \times G \rightarrow M$, while the correct construction by the homotopy $\operatorname{limit}$ holim $B G^{\mathrm{con}}\left(U_{\mathbf{0}}\right) \in$ Grpd is much richer as it captures all possible principal $G$-bundles on $M$. We also refer to $[29,57]$ for a more philosophical perspective on gauge fields, groupoids and aspects of richness.

\subsection{The role of stacks}

The groupoid perspective on gauge theories that we have introduced in the previous section is incomplete because it neglects the smooth structure on spaces of gauge fields. We shall now explain how the concept of stacks resolves this issue. We refer to [48] for technical details on the model categorical approach to 1-stacks that we review below, and to [31] for analogous developments for $\infty$-stacks. We also refer to [55] for an $\infty$-categorical approach to stacks and to [61] for a broad overview, including applications to physics.

The way how stacks formalize smooth structures is more abstract than the standard approach adopted in differential geometry, which amounts to endowing a space with an atlas of charts. In order to illustrate the basic ideas, let us first explain how one can describe manifolds from such a more abstract perspective. Let Man be the category of (finite-dimensional) manifolds and smooth maps. Instead of describing a manifold $M \in$ Man by looking for suitable charts, we shall study the sets $C^{\infty}(T, M) \in$ Set of smooth maps from all test manifolds $T \in$ Man into $M$. Note that these sets capture a lot of (in fact, all) information about the manifold $M$, for example:

- $C^{\infty}\left(\mathbb{R}^{0}, M\right)$ describes the points in $M$,

- $C^{\infty}\left(\mathbb{R}^{1}, M\right)$ describes the smooth curves in $M$, and

- $C^{\infty}\left(\mathbb{R}^{2}, M\right)$ describes the smooth surfaces in $M$, etc.

In particular, the sets $C^{\infty}(T, M)$ see the smooth structure on $M$. Observe that the assignment $T \mapsto C^{\infty}(T, M)$ is contravariantly functorial, i.e. it defines a functor $C^{\infty}(-, M)$ : 
Man $^{\text {op }} \rightarrow$ Set to the category of sets. This is called the functor of points of the manifold $M$. Because smooth functions between manifolds can be glued, we further observe that $C^{\infty}(-, M) \in \mathbf{S h}(\mathbf{M a n})$ is a sheaf on the site of manifolds Man with the usual open cover Grothendieck topology. As a consequence of the Yoneda Lemma, the assignment

$$
\operatorname{Man} \longrightarrow \mathbf{S h}(\operatorname{Man}), M \longmapsto C^{\infty}(-, M)
$$

is a fully faithful functor, i.e. smooth maps $M \rightarrow N$ between two manifolds can be identified with natural transformations $C^{\infty}(-, M) \rightarrow C^{\infty}(-, N)$ between their functors of points. Finally, because each manifold admits a good open cover, the category $\mathbf{S h}(\operatorname{Man})$ of sheaves on Man is equivalent to the category $\mathbf{S h}(\mathbf{C a r t})$ of sheaves on the site of Cartesian spaces Cart with the good open cover Grothendieck topology.

Summing up, we observed that the category Man of manifolds can be identified with a full subcategory

$$
\operatorname{Man} \subseteq \mathbf{H}_{0}:=\operatorname{Sh}(\text { Cart })
$$

of the category of Set-valued sheaves on the site of Cartesian spaces Cart. This means that one can equivalently study manifolds and smooth maps between manifolds from the perspective of their functors of points. In particular, the smooth structure of a manifold $M$ is encoded entirely in the smooth mappings $\mathbb{R}^{n} \rightarrow M$ from general test spaces $\mathbb{R}^{n}$, for $n \geq 0$, into $M$.

Note that the category $\mathbf{H}_{0}$ is "vastly bigger" than the category of manifolds Man. Objects $X \in \mathbf{H}_{0}$ that are not (isomorphic to) manifolds should be thought of as generalized smooth spaces, where the smooth structure is encoded, in the spirit of functors of points, by the sets of $\mathbf{H}_{0}$-morphisms $\mathbb{R}^{n} \rightarrow X$, for all $n \geq 0$. There are plenty of interesting generalized smooth spaces that feature in field theory. The following is a small list of concrete examples.

Example 3.6. Let $M$ and $N$ be two manifolds, which we regard as objects in $\mathbf{H}_{0}$. Because $\mathbf{H}_{0}$ is a Cartesian closed category (even better, it is a topos), one can form the internal hom object $[M, N] \in \mathbf{H}_{0}$. This is a generalized smooth space that describes the space of smooth mappings from $M$ to $N$. Why is that so? To answer this question, let us first look at the points $\mathbb{R}^{0} \rightarrow[M, N]$ of this generalized smooth space. Using that $[M,-]$ is the right adjoint functor of $(-) \times M$, we can compute the set of points via

$$
\begin{aligned}
& \operatorname{Hom}_{\mathbf{H}_{0}}\left(\mathbb{R}^{0},[M, N]\right) \cong \operatorname{Hom}_{\mathbf{H}_{0}}\left(\mathbb{R}^{0} \times M, N\right) \\
& \cong \operatorname{Hom}_{\mathbf{H}_{0}}(M, N) \cong C^{\infty}(M, N),
\end{aligned}
$$

where in the last step we used that the inclusion Man $\rightarrow$ $\mathbf{H}_{0}$ is fully faithful. Thus, the underlying set of points of
$[M, N]$ is precisely the set of all smooth maps from $M$ to $N$. In order to get some feeling for the smooth structure of $[M, N]$, we note that a similar computation shows that

$\operatorname{Hom}_{\mathbf{H}_{0}}\left(\mathbb{R}^{n},[M, N]\right) \cong C^{\infty}\left(\mathbb{R}^{n} \times M, N\right)$.

In particular, the smooth curves $\mathbb{R}^{1} \rightarrow[M, N]$ are precisely the smooth functions $\mathbb{R}^{1} \times M \rightarrow N$, which matches the naive expectation for a smooth structure on a mapping space. We refer to [9] for an application of topos theoretic techniques to non-linear field theories.

Example 3.7. For $p \geq 0$, consider the functor $\Omega^{p}$ : Cart $^{\text {op }} \rightarrow$ Set, $\mathbb{R}^{n} \mapsto \Omega^{p}\left(\mathbb{R}^{n}\right)$ that assigns $p$-forms to Cartesian spaces. This functor defines a sheaf on Cart and hence a generalized smooth space $\Omega^{p} \in \mathbf{H}_{0}$. This space is called the classifying space of $p$-forms because $\mathbf{H}_{0^{-}}$morphisms $M \rightarrow \Omega^{p}$ from a manifold into this space correspond precisely to $p$-forms on $M$. Let us provide the relevant argument: For $M=\mathbb{R}^{n}$, this is a direct consequence of the Yoneda Lemma. For a general manifold $M$, one chooses any good open cover $\left\{U_{i} \subseteq M\right\}$ and uses that $\operatorname{colim}\left(\amalg_{i j} U_{i j} \rightrightarrows \amalg_{i} U_{i}\right) \rightarrow M$ is an isomorphism in $\mathbf{H}_{0}$. It follows that a morphism $M \rightarrow \Omega^{p}$ is precisely a family of $p$-forms $\omega_{i} \in \Omega^{p}\left(U_{i}\right)$ satisfying $\left.\omega_{i}\right|_{U_{i j}}=\left.\omega_{j}\right|_{U_{i j}}$, for all $i, j$. The sheaf property of $p$-forms then implies that this data can be glued to a single $p$-form $\omega \in \Omega^{p}(M)$ on the manifold $M$. As a side-remark, we would like to mention that the classifying space $\Omega^{p} \in \mathbf{H}_{0}$ can be used to define a concept of $p$-forms on any generalized smooth space $X \in \mathbf{H}_{0}$ in terms of $\mathbf{H}_{0}$-morphisms $X \rightarrow \Omega^{p}$. For example, a $p$-form on the mapping space $[M, N] \in \mathbf{H}_{0}$ from Example 3.6 is simply an $\mathbf{H}_{0}$-morphism $[M, N] \rightarrow \Omega^{p}$.

Let us now turn our attention to stacks. Loosely speaking, a stack resembles a generalized smooth space in the sense above, however with the crucial difference that its functor of points is valued in Grpd instead of Set. (Recall from the previous section that groupoids play a fundamental role in gauge theory.) In contrast to the strict sheaf condition for generalized smooth spaces in $\mathbf{H}_{0}$, stacks satisfy a weaker homotopy sheaf condition. More precisely, we have the following definition [48].

Definition 3.8. A stack is a presheaf of groupoids $X$ : Cart $^{\mathrm{op}} \rightarrow$ Grpd that satisfies the homotopy sheaf condition: For each $U \in$ Cart and good open cover $\left\{U_{i} \subseteq U\right\}$, the canonical map

$$
X(U) \stackrel{\sim}{\longrightarrow} \operatorname{holim}\left(\prod_{i} X\left(U_{i}\right) \rightrightarrows \prod_{i j} X\left(U_{i j}\right) \rightrightarrows \cdots\right)
$$

is a weak equivalence in the model category Grpd, where holim is the homotopy limit of a cosimplicial groupoid (cf. Lemma 3.3). 
It was shown in [48] that stacks are (the fibrant) objects in a suitable model category $\mathbf{H}_{1}$. Let us briefly explain this crucial point without going too much into the details. As a first step, let us consider the category PSh(Cart, Grpd) of groupoid-valued presheaves on Cart. The model structure on Grpd induces the projective model structure on this functor category, i.e. a morphism $\zeta: X \rightarrow Y$ is a fibration (respectively, a weak equivalence) if all components $\zeta_{U}: X(U) \rightarrow Y(U)$, for $U \in$ Cart, are fibrations (respectively, weak equivalences) in Grpd. This model structure is however not yet quite right, because it does not take into account the Grothendieck topology on Cart. Given any good open cover $\left\{U_{i} \subseteq U\right\}$ of some $U \in$ Cart, we form its Čech nerve as in (3.8), which defines a simplicial object $U_{\bullet} \in \mathbf{P S h}(\text { Cart, Grpd) })^{\Delta^{\mathrm{op}}}$ by regarding $U_{i_{1} \cdots i_{n}} \in$ PSh(Cart, Grpd) via the Yoneda embedding. One then defines the model category

$\mathbf{H}_{1}:=\operatorname{PSh}(\text { Cart, Grpd })_{\text {loc }}$

by left Bousfield localization of the projective model structure at the set of morphisms

$\left\{U \longleftarrow \operatorname{hocolim} U_{\bullet}:\left\{U_{i} \subseteq U\right\}\right.$ good open cover $\}$,

where hocolim : PSh $(\text { Cart, Grpd })^{\Delta^{\text {op }}} \rightarrow \mathbf{P S h}($ Cart, Grpd) is the homotopy colimit with respect to the projective model structure on PSh(Cart, Grpd). The relationship between stacks and $\mathbf{H}_{1}$ is explained in the following proposition, which was proven in [48].

Proposition 3.9. Stacks according to Definition 3.8 are precisely the fibrant objects in $\mathbf{H}_{1}$.

Example 3.10. Each manifold $M \in$ Man defines a stack by composing its functor of points $C^{\infty}(-, M):$ Cart $^{\mathrm{op}} \rightarrow$ Set with the inclusion Set $\rightarrow$ Grpd. We shall denote the stack corresponding to a manifold simply by $M \in \mathbf{H}_{1}$. More generally, we have an inclusion $\mathbf{H}_{0} \rightarrow \mathbf{H}_{1}$ of the category of generalized smooth spaces into $\mathbf{H}_{1}$ that takes values in stacks.

Example 3.11. Recall from Example 3.2 the presheaf of groupoids $B G^{\text {con }}:$ Cart $^{\text {op }} \rightarrow$ Grpd. From the calculation in Example 3.5, it follows that this defines a stack $B G^{\text {con }} \in \mathbf{H}_{1}$, which is called the classifying stack of principal $G$-bundles with connections. This requires some further explanations. Let $M$ be a manifold, regarded as an object $M \in \mathbf{H}_{1}$. Computing the naive groupoid $\operatorname{hom}_{\mathbf{H}_{1}}\left(M, B G^{\mathrm{con}}\right) \in \mathbf{G r p d}$ of $\mathbf{H}_{1}$-morphisms $M \rightarrow B G^{\text {con }}$ (see e.g. [11]), one obtains the groupoid whose objects are $A \in \Omega^{1}(M, \mathfrak{g})$ and morphisms are gauge transformations $A \rightarrow A \triangleleft h$, for $h \in C^{\infty}(M, G)$.
At first sight that seems very strange, because the latter groupoid does not describe non-trivial principal $G$ bundles on $M$ and hence the name classifying space for $B G^{\text {con }}$ seems unjustified. So what went wrong? It turns out that computing the groupoids of $\mathbf{H}_{1}$-morphisms hom $_{\mathbf{H}_{1}}: \mathbf{H}_{1}^{\mathrm{op}} \times \mathbf{H}_{1} \rightarrow$ Grpd is one of the (many) instances where derived functors are crucial. So what went wrong is that we forgot to derive this functor! Because $B G^{\text {con }} \in \mathbf{H}_{1}$ is a fibrant object by Proposition 3.9, a model for the derived groupoid of $\mathbf{H}_{1}$-morphisms is given by $\mathbb{R h o m}_{\mathbf{H}_{1}}\left(M, B G^{\mathrm{con}}\right)=\operatorname{hom}_{\mathbf{H}_{1}}\left(Q M, B G^{\text {con }}\right)$, where $Q M \rightarrow$ $M$ is a cofibrant replacement of the manifold $M$ in $\mathbf{H}_{1}$. Using as in [11, Appendix B] a good open cover $\left\{U_{i} \subseteq M\right\}$ to define a cofibrant replacement of $M$, one immediately realizes that $\mathbb{R h o m}_{\mathbf{H}_{1}}\left(M, B G^{\mathrm{con}}\right) \in \mathbf{G r p d}$ can be computed precisely as the homotopy limit of the cosimplicial groupoid displayed in (3.9). Therefore, recalling Example 3.5, $\mathbb{R h o m}_{\mathbf{H}_{1}}\left(M, B G^{\text {con }}\right) \in \mathbf{G r p d}$ is the correct groupoid of all principal $G$-bundles with connections on $M$ (together with their gauge transformations), eventually justifying the interpretation of $B G^{\text {con }}$ as classifying stack of principal $G$-bundles with connections.

Example 3.12. Our original aim of this section was to describe a smooth structure on the groupoids of gauge fields. This can now be achieved by working within the framework of stacks that we discussed above. Let us briefly review how a moduli stack of gauge fields can be constructed by performing (derived functorial) constructions in the model category $\mathbf{H}_{1}$. As input data, we choose any manifold $M$ (on which the gauge fields should live) and any Lie group $G$ (the structure group of the gauge theory). Recalling the previous two examples, we obtain the two stacks $M \in \mathbf{H}_{1}$ and $B G^{\text {con }} \in \mathbf{H}_{1}$. Using that $\mathbf{H}_{1}$ is a Cartesian closed model category (even better, it is a higher topos), one can form the derived internal hom object $\mathbb{R}\left[M, B G^{\mathrm{con}}\right] \in \mathbf{H}_{1}$, which one should interpret similarly to Example 3.6 as a stack of mappings from $M$ to $B G^{\mathrm{con}}$. From Example 3.11, we know that the groupoid of points $\mathbb{R}^{0} \rightarrow \mathbb{R}\left[M, B G^{\text {con }}\right]$ is the groupoid of all principal $G$-bundles with connections on $M$, i.e. the groupoid of all gauge fields. Unfortunately, the smooth structure on the mapping stack $\mathbb{R}\left[M, B G^{\mathrm{con}}\right]$ is not the desired one, because, as one can show by a direct computation (cf. [11]), a smooth curve $\mathbb{R}^{1} \rightarrow \mathbb{R}\left[M, B G^{\text {con }}\right]$ is given by a principal $G$-bundle with connection on the product manifold $\mathbb{R}^{1} \times M$ and not by an $\mathbb{R}^{1}$-parametrized family of principal $G$-bundles with connections on $M$. The solution to this issue that was proposed in [61] and refined in [11] is to perform a differential concretification of the mapping stack $\mathbb{R}\left[M, B G^{\text {con }}\right]$. Loosely speaking, this is a model categorical construction that "kills off" the bundles and connections 
on the test spaces $\mathbb{R}^{n} \in$ Cart. (This can be interpreted in terms of vertical geometry with respect to the projection $\mathrm{pr}_{1}: \mathbb{R}^{n} \times M \rightarrow \mathbb{R}^{n}$ onto test spaces.) As this construction is quite technical, we refer to the original papers for the details. This defines a new stack $\mathfrak{C o n}_{G}(M) \in \mathbf{H}_{1}$, called the differential concretification of $\mathbb{R}\left[M, B G^{\mathrm{con}}\right] \in \mathbf{H}_{1}$, which describes our desired moduli stack of principal $G$-bundles with connections on a manifold $M$. This construction is functorial in the sense that $\mathfrak{C o n}_{G}: \mathbf{M a n}^{\text {op }} \rightarrow \mathbf{H}_{1}$ defines a functor taking values in stacks. (Strictly speaking, this requires the choice of a functorial cofibrant replacement for manifolds, e.g. the one in [11, Appendix B].) As a last remark, we would like to add that moduli stacks of solutions to, e.g., the non-Abelian Yang-Mills equation or the Chern-Simons equation can also be constructed from such a perspective. See [11] for the details on Yang-Mills theory and [35] for Chern-Simons theory.

Remark 3.13. We conclude this section by briefly commenting on how to describe $\infty$-stacks from a model categorical perspective. See e.g. [30,31] for the details. Let us recall that an explicit model for the $\infty$-category $\infty$ Grpd is given by endowing the category sSet $=$ Set $^{\Delta^{\text {op }}}$ of simplicial sets with the usual Kan-Quillen model structure. The fibrant objects in this model category are the Kan complexes, which are a model for $\infty$-groupoids. Instead of Grpd-valued presheaves on Cart, the description of $\infty$-stacks starts from the category PSh(Cart, sSet) of presheaves with values in sSet. The left Bousfield localization of the projective model structure at all hypercovers defines the model category

$\mathbf{H}_{\infty}:=\operatorname{PSh}(\text { Cart, } \mathbf{s S e t})_{\text {loc }}$.

$\infty$-stacks are then by definition the fibrant objects in $\mathbf{H}_{\infty}$. Similarly to Definition 3.8 and Proposition $3.9, \infty$-stacks can be characterized by a suitable homotopy sheaf condition with respect to hypercovers. Finally, the inclusions from Remark 3.1 generalize to

$\mathbf{H}_{0} \hookrightarrow \mathbf{H}_{1} \hookrightarrow \mathbf{H}_{2} \hookrightarrow \cdots \hookrightarrow \mathbf{H}_{\infty} \quad$,

which means that all generalized smooth spaces, stacks, 2-stacks,..., can be regarded as particular examples of $\infty$-stacks.

\subsection{Smooth cochain algebras on stacks}

In the previous sections we have seen that higher structures are crucial for the description of "spaces" of gauge fields, which are in fact higher categorical spaces called stacks. Thinking ahead towards QFT, which requires a concept of observable algebras, we would like to explain what it means to form "function algebras" on stacks. Our statements below are formulated for the more general case of $\infty$-stacks in $\mathbf{H}_{\infty}$, because this does not lead to any further complications compared to the case of 1-stacks in $\mathbf{H}_{1}$.

Before explaining our concept of smooth cochain algebras on $\infty$-stacks, we would like to start with the related, but simpler, case of cochain algebras on simplicial sets. See e.g. [15] for a more extensive review. In the following $k$ will be a field of characteristic 0 , e.g. $k=\mathbb{R}$ or $k=\mathbb{C}$, and $\mathbf{C h}(k)$ the symmetric monoidal model category of (possibly unbounded) chain complexes of $k$-vector spaces, see e.g. [50]. Let us recall that in this model structure a morphism $f: V \rightarrow W$ between two chain complexes is

- a weak equivalence if it is a quasi-isomorphism, i.e. it induces an isomorphism $H_{\bullet}(f): H_{\bullet}(V) \rightarrow H_{\bullet}(W)$ in homology;

- a fibration if it is degree-wise surjective;

- a cofibration if it has the left lifting property with respect to all acyclic fibrations.

Recall that to every simplicial set $S \in$ sSet, one can associate the chain complex $N_{*}(S, k) \in \mathbf{C h}(k)$ of normalized $k$ valued chains on $S$. The functor $N_{*}(-, k): \mathbf{s S e t} \rightarrow \mathbf{C h}(k)$ is the left adjoint of a Quillen adjunction between the model category sSet, with the Kan-Quillen model structure, and the model category $\mathbf{C h}(k)$. Composing $N_{*}(-, k)$ with the internal hom functor $[-, k]: \mathbf{C h}(k) \rightarrow \mathbf{C h}(k)^{\text {op }}$ for chain complexes, which is also a left Quillen functor, defines a left Quillen functor $N^{*}(-, k): \mathbf{s S e t} \rightarrow \mathbf{C h}(k)^{\text {op }}$ that assigns to a simplicial set its normalized $k$-valued cochains. By [16], the latter are canonically $E_{\infty}$-algebras, i.e. homotopycoherently commutative differential graded algebras. (We refer to Section 4 for more details on homotopy algebras over operads.) Summing up, we obtained a left Quillen functor

$N^{*}(-, k): \mathbf{s S e t} \longrightarrow \operatorname{Alg}_{\mathscr{E}_{\infty}}(\mathbf{C h}(k))^{\text {op }}$

that assigns to each simplicial set $S \in$ sSet its normalized cochain algebra $N^{*}(S, k) \in \operatorname{Alg}_{\mathscr{E}_{\infty}}(\mathbf{C h}(k))$. Because all simplicial sets are cofibrant in the Kan-Quillen model structure, this functor preserves weak equivalences and does not have to be derived.

Example 3.14. Every set $S \in$ Set can be regarded as a constant simplicial set that we also denote by $S \in \mathbf{s S e t}$. The normalized cochain algebra in this case is a chain complex concentrated in degree 0 with trivial differential, i.e. it is just a vector space. A concrete calculation shows that $N^{*}(S, k)=\operatorname{Map}(S, k)$ is the usual commutative algebra of $k$-valued functions on the set $S$.

Example 3.15. More interestingly, let $\mathfrak{G} \in$ Grpd be a groupoid and consider its nerve $B \mathfrak{G} \in$ sSet. Then the normalized cochain algebra $N^{*}(B \mathfrak{G}, k)$ is precisely the 
usual $k$-valued groupoid cohomology dg-algebra, see e.g. [27]. Note that this is in general not a strictly commutative dg-algebra, but an $E_{\infty}$-algebra. Thinking of $\mathfrak{G} \in \mathbf{G r p d}$ as a groupoid of gauge fields, the dg-algebra $N^{*}(B \mathfrak{G}, k)$ describes both functions of gauge fields (in degree 0 ) and functions of ghost fields (in homological degree $<0$, or in cohomological degree $>0)$. In fact, $N^{*}(B \mathfrak{G}, k)$ is a groupoid version of the usual Chevalley-Eilenberg dgalgebra from Lie algebroid cohomology.

The construction of cochain algebras above can be generalized to the case of $\infty$-stacks. We shall provide a brief sketch and refer to [15] for the technical details. Let us consider for the moment the case where all presheaf categories (with values in model categories) are endowed with the projective model structures. Applying the normalized chain functor $N_{*}(-, k)$ : sSet $\rightarrow \mathbf{C h}(k)$ object-wise on presheaves defines a left Quillen functor that we denote with abuse of notation by the same symbol

$N_{*}(-, k): \mathbf{P S h}($ Cart, sSet $) \longrightarrow \operatorname{PSh}($ Cart, $\mathbf{C h}(k)) \quad$.

Concretely, given a presheaf of simplicial sets $X:$ Cart $^{\mathrm{op}} \rightarrow$ sSet, then $N_{*}(X, k):$ Cart $^{\mathrm{op}} \rightarrow \mathbf{C h}(k)$ is the presheaf of chain complexes defined by $N_{*}(X, k)(U):=N_{*}(X(U), k) \in$ $\mathbf{C h}(k)$, for all $U \in$ Cart. Assuming in the following that $k=\mathbb{R}$ or $k=\mathbb{C}$, we can promote $k$ to an object $\underline{k} \in$ $\operatorname{PSh}(\operatorname{Cart}, \mathbf{C h}(k))$ by setting $\underline{k}(U):=C^{\infty}(U, k)$ to be the vector space of $k$-valued smooth functions, for all $U \in$ Cart. Because PSh $(\operatorname{Cart}, \mathbf{C h}(k))$ is enriched over $\mathbf{C h}(k)$ and $k$ is a fibrant object, there is a left Quillen functor

$[-, \underline{k}]^{\infty}: \operatorname{PSh}(\operatorname{Cart}, \mathbf{C h}(k)) \longrightarrow \mathbf{C h}(k)^{\text {op }}$

that assigns chain complexes of morphisms. This functor should be understood as taking smooth $k$-valued functions. Concretely, given any object $V \in \mathbf{P S h}(\mathbf{C a r t}, \mathbf{C h}(k))$, one has an explicit description by an end

$[V, \underline{k}]^{\infty}=\int_{U \in \operatorname{Cart}^{\text {op }}}[V(U), \underline{k}(U)]$,

where [-, - denotes the internal hom functor in $\mathbf{C h}(k)$. Composing (3.20) and (3.21) defines a left Quillen functor

$N^{\infty *}(-, k): \mathbf{P S h}($ Cart, sSet $) \longrightarrow \operatorname{Alg}_{\mathscr{E}_{\infty}}(\mathbf{C h}(k))^{\text {op }}$

that assigns $E_{\infty}$-algebras because of [16]. We shall call this the smooth normalized cochain algebra functor.

Proposition 3.16. The left derived functor $\mathbb{\square} N^{\infty *}(-, k)$ of (3.23) restricts to a homotopical functor on the full subcategory $\mathbf{S t}_{\infty} \subseteq \mathbf{H}_{\infty}$ of $\infty$-stacks (the fibrant objects in $\mathbf{H}_{\infty}$ ), i.e.

$\mathbb{L} N^{\infty *}(-, k): \mathbf{S t}_{\infty} \longrightarrow \operatorname{Alg}_{\mathscr{E}_{\infty}}(\mathbf{C h}(k))^{\mathrm{op}}$

is a functor that preserves weak equivalences between $\infty$ stacks.
Remark 3.17. In contrast to normalized cochain algebras on simplicial sets, the functor (3.23) must be derived because not every presheaf of simplicial sets is a cofibrant object. A relatively concrete model for cofibrant replacement in this case is given by Dugger in [30].

Example 3.18. Let $\mathbf{C}$ be a category (of spacetimes) and $\mathfrak{F}: \mathbf{C}^{\mathrm{op}} \rightarrow \mathbf{S t}_{\infty} \subseteq \mathbf{H}_{\infty}$ a functor that assigns to each spacetime $c \in \mathbf{C}$ an $\infty$-stack $\mathfrak{F}(c)$ of gauge fields on $c$. For example, this could be the functor $\mathfrak{C o n}_{G}: \mathbf{M a n}^{\mathrm{op}} \rightarrow \mathbf{S t}_{\infty}$ assigning the moduli stacks of principal $G$-bundles with connections (cf. Example 3.12) or the functor $\mathfrak{Y M M}_{G}:$ Loc $^{\mathrm{op}} \rightarrow$ $\mathbf{S t}_{\infty}$ assigning the moduli stacks of solutions of the YangMills equation (cf. [11]). Applying (3.24) defines for each spacetime $c \in \mathbf{C}$ an $E_{\infty}$-algebra

$\mathfrak{A}(c):=\mathbb{L} N^{\infty *}(\mathfrak{F}(c), k) \in \operatorname{Alg}_{\mathscr{E}_{\infty}}(\mathbf{C h}(k))$

that one should interpret as a classical observable algebra for the gauge fields on $c$. Functoriality of this construction implies that $\mathfrak{A}: \mathbf{C} \rightarrow \mathbf{A l g}_{\mathscr{E}_{\infty}}(\mathbf{C h}(k))$ is a covariant functor on $\mathbf{C}$, which is very similar to the structures considered in AQFT, cf. Section 2. There are however two main differences: 1.) The observable algebras that are assigned here are dg-algebras, i.e. associative and unital algebras in chain complexes, not ordinary algebras in vector spaces. The higher structures in the $\infty$-stacks of gauge fields are represented by higher homology groups of these dg-algebras. 2.) Even though no quantization happened so far, the observable algebras are not strictly commutative, but commutative up to coherent homotopies. Therefore, in order to understand gauge theories in AQFT, one is naturally lead to consider homotopy-coherent algebraic structures. This will be formalized in Section 4 .

Open Problem 3.19. For applications to gauge theory, the $\infty$-stacks $X$ typically carry a Poisson structure (or symplectic structure) which is determined by the action functional. It is currently unclear to us how one can construct (in a homotopically meaningful way) a Poisson bracket on the $E_{\infty}$-algebra $\llbracket N^{\infty *}(X, k)$. It is even more unclear to us how one can quantize (in a homotopically meaningful way) such homotopy-coherent versions of Poisson dgalgebras, which is required for constructing examples of quantum gauge theories. Quite recently there have been impressive developments in derived algebraic geometry $[24,58]$ that focus on related questions and we hope to see some fruitful interplay with this discipline in the future.

\subsection{Derived geometry of linear gauge fields}

There is a second kind of higher structures in (gauge) field theory that has a different origin than the groupoid (or $\infty$ - 
groupoid) structures discussed in the previous sections. Summarizing the latter in a single sentence, groupoids and stacks become important whenever one divides out gauge symmetries that usually do not act freely on the gauge fields. (Recall the role of stabilizers at the beginning of Section 3.1.) In a (gauge) field theory one typically starts from a space or stack $\mathfrak{F}$ of fields together with an action functional $S: \mathfrak{F} \rightarrow \mathbb{R}$. The aim is then to describe the space of solutions of the corresponding Euler-Lagrange equations, which can be obtained from the following construction: First, one considers the variation of the action $S$, which defines a section $\mathrm{d} S: \mathfrak{F} \rightarrow T^{*} \mathfrak{F}$ of the cotangent bundle over the space (or stack) of fields. Informally, the space of solutions is the "subspace" $\mathfrak{S o l} \subseteq \mathfrak{F}$ on which $\mathrm{d} S: \mathfrak{F} \rightarrow T^{*} \mathfrak{F}$ coincides with zero section $0: \mathfrak{F} \rightarrow T^{*} \mathfrak{F}$. This is formalized by forming the (homotopy) pullback

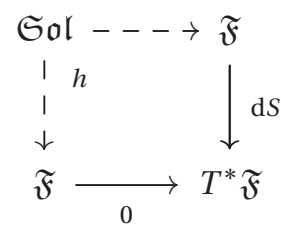

in the appropriate (model) category of spaces or stacks. Geometrically, one can interpret this construction as computing the intersection of $\mathrm{d} S$ with the zero section 0 .

For a generic action functional $S$, the intersection in (3.26) will be far away from being transversal and hence the space (or stack) Sol can be badly behaved. Furthermore, it ignores more refined information about the intersection problem, such as the multiplicities of multiple intersections. A solution to these problems is proposed by derived algebraic geometry $[24,58]$, where a more refined concept of spaces, called derived $\infty$-stacks, is developed.

Let us explain very briefly the basic idea behind derived $\infty$-stacks, without going into any technical details. Recall from Section 3.2 that an $\infty$-stack is described by its functor of points $X$ : Cart $^{\mathrm{op}} \rightarrow$ sSet that assigns to each Cartesian space $U \in$ Cart the $\infty$-groupoid $X(U)$ of points of shape $U$ in $X$. Recall that the latter encode both the gauge fields and the (higher) gauge symmetries. A derived $\infty$-stack is a more refined concept that is described by a functor of points of the form $X:$ cCart $^{\text {op }} \rightarrow$ sSet, where cCart $=$ Cart $^{\Delta}$ are cosimplicial test spaces. (In algebraic geometry, these are described by the opposite category of simplicial commutative $k$-algebras $\mathbf{s C A l g}_{k}$, cf. [24,58].) Note that there are two opposite degrees appearing in a derived $\infty$-stack: The "stacky" simplicial degree in the target category sSet and the "derived" cosimplicial degree in the source category cCart. Morally speaking, the former encodes refined aspects of gauge symmetries and the latter encodes refined aspects of intersections. We will later see that these two different degrees are related to ghost fields and anti fields in the BRST/BV formalism.

Working with derived $\infty$-stacks is very hard. In particular, we are not yet able to describe physically interesting examples of solutions spaces of gauge theories within this approach. (Toy-models of such are discussed in $[24,58]$.) In what follows we shall focus on a certain approximation of the structures appearing in derived algebraic geometry, which however encodes some of the crucial features of this approach. Let us motivate this approximation. As mentioned above, derived $\infty$-stacks come with two degrees, "stacky" degrees in sSet and "derived" degrees in cCart. If one restricts to linear spaces and linear maps between them, the Dold-Kan correspondence allows us to describe the "stacky" degrees by non-negatively graded chain complexes $\mathbf{C h}_{\geq 0}(k)$ and the "derived" degrees by non-positively graded chain complexes $\mathbf{C h}_{\leq 0}(k)$. Our working assumption below is thus that unbounded chain complexes $\mathbf{C h}(k)$ capture linear features of derived $\infty$-stacks. A similar perspective is taken in the work of Costello and Gwilliam [26].

Let us now focus on a very simple example to illustrate the main features of derived $\infty$-stacks in field theory. In what follows we fix $k=\mathbb{R}$ and take any oriented and timeoriented globally hyperbolic Lorentzian manifold $M \in$ Loc, interpreted as spacetime. We consider Abelian gauge fields with structure group $G=\mathbb{R}$. Because there are no non-trivial principal $\mathbb{R}$-bundles, the groupoid of gauge fields (cf. Example 3.2) on $M$ is given by

$$
B G^{\mathrm{con}}(M)=\left\{\begin{array}{ll}
\text { Obj: } & A \in \Omega^{1}(M) \\
\text { Mor: } & A \stackrel{\epsilon}{\longrightarrow} A+\mathrm{d} \epsilon \\
& \text { with } \epsilon \in C^{\infty}(M)
\end{array} .\right.
$$

Following [12], one easily computes the nerve of this groupoid and, after applying the Dold-Kan correspondence to the resulting simplicial vector space, obtains the chain complex

$$
\mathfrak{F}=\left(\stackrel{(0)}{\Omega^{1}(M) \stackrel{\mathrm{d}}{\leftarrow} \Omega^{(1)}(M)}\right)
$$

where we indicated in round brackets the homological degrees and identified functions with 0 -forms $\Omega^{0}(M)=$ $C^{\infty}(M)$. As expected, this chain complex has only "stacky" positive degrees and no "derived" negative degrees.

As action functional we take the ordinary Abelian YangMills action on the spacetime $M$, i.e. $S=\int_{M} \frac{1}{2} \mathrm{~d} A \wedge * \mathrm{~d} A$, where $*$ is the Hodge operator. A naive variation of this action leads to the Abelian Yang-Mills equation $\delta \mathrm{d} A=0$, where $\delta$ is the codifferential, i.e. the formal adjoint of $\mathrm{d}$ 
with respect to the inner product $\langle\omega, \lambda\rangle=\int_{M} \omega \wedge * \lambda$ on $p$ forms. We would like to describe the space of solutions of the Abelian Yang-Mills equation from the perspective of derived geometry by computing a homotopy pullback as in (3.26), which is also called the derived critical locus. For this we have to introduce a cotangent bundle $T^{*} \mathfrak{F}$ over the space of fields $\mathfrak{F}$. In line with our working assumption that $\mathfrak{F} \in \mathbf{C h}(k)$ models a "linear space" (in the sense of being a chain complex of $k$-vector spaces), it is reasonable to define the cotangent bundle

$T^{*} \mathfrak{F}:=\mathfrak{F} \times \mathfrak{F}^{*} \in \mathbf{C h}(k)$

as the product in $\mathbf{C h}(k)$ of the chain complex of fields and an appropriate choice of linear dual of that. Choosing the "smooth dual"

$\mathfrak{F}^{*}:=\left(\begin{array}{ccc}(-1) & \stackrel{-\delta}{(0)} \\ \Omega^{0}(M) & \leftarrow & \Omega^{1}(M)\end{array}\right)$,

one obtains

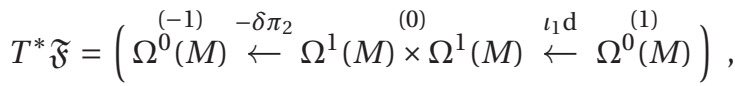

where $\iota_{1}: \Omega^{1}(M) \rightarrow \Omega^{1}(M) \oplus \Omega^{1}(M)=\Omega^{1}(M) \times \Omega^{1}(M)$ is the inclusion of the first factor and $\pi_{2}: \Omega^{1}(M) \times \Omega^{1}(M) \rightarrow$ $\Omega^{1}(M)$ the projection on the second factor.

Open Problem 3.20. Note that the "smooth dual" $\mathfrak{F}^{*}$ we have chosen in (3.30) is not obtained via the categorical concept of dual chain complexes. In fact, the dualizable objects in $\mathbf{C h}(k)$ are perfect complexes, i.e. chain complexes that are quasi-isomorphic to a bounded chain complex of finite-dimensional $k$-vector spaces, however $\mathfrak{F} \in \mathbf{C h}(k)$ is clearly not perfect. As a consequence, it is presently not clear to us if the construction of the cotangent bundle $T^{*} \mathfrak{F}$ preserves weak equivalences in $\mathbf{C h}(k)$. According to our best knowledge, it is an open problem how to formalize a model categorical (or higher categorical) concept of "smooth duals" as in (3.30).

The variation of the action $S$ defines a $\mathbf{C h}(k)$-morphism $\mathrm{d} S: \mathfrak{F} \rightarrow T^{*} \mathfrak{F}$ that is concretely given by

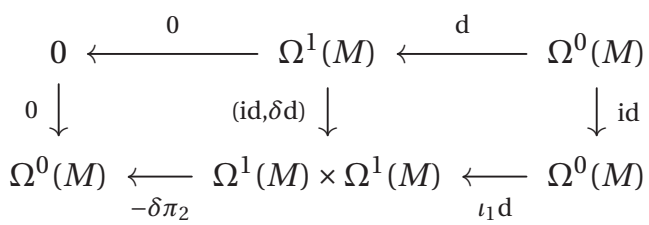

Moreover, the zero section $0: \mathfrak{F} \rightarrow T^{*} \mathfrak{F}$ is the $\mathbf{C h}(k)$ morphism given by

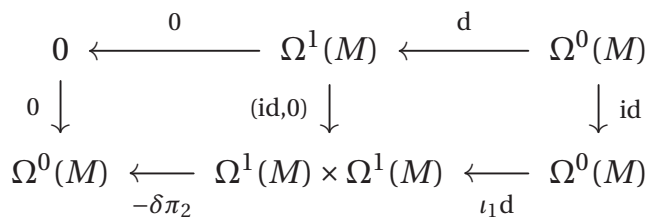

Proposition 3.21. Consider as above Abelian Yang-Mills theory with structure group $G=\mathbb{R}$ on a spacetime $M \in \mathbf{L o c}$. A model for the corresponding homotopy pullback (3.26) in $\mathbf{C h}(k)$ is given by

Sol $=\left(\Omega^{(-2)}(M) \stackrel{\delta}{\leftarrow} \Omega^{(1)}(M) \stackrel{\delta \mathrm{d}}{\leftarrow} \Omega^{10}(M) \stackrel{\mathrm{d}}{\leftarrow} \Omega^{(1)}(M)\right)$.

Proof. By [47, Corollary 13.1.3], $\mathbf{C h}(k)$ is a right proper model category because each object is fibrant. As a consequence of [47, Corollary 13.3.8], one can compute the homotopy pullback (3.26) in terms of an ordinary pullback if we replace the zero section $0: \mathfrak{F} \rightarrow T^{*} \mathfrak{F}$ by a weakly equivalent fibration. Because the zero section is the Cartesian product of the $\mathbf{C h}(k)$-morphisms id $: \mathfrak{F} \rightarrow \mathfrak{F}$ and $0: 0 \rightarrow \mathfrak{F}^{*}$, the problem reduces to finding a fibration that is weakly equivalent to the zero map $0: 0 \rightarrow \mathfrak{F}^{*}$.

For this let us introduce the chain complex

$D:=\left(\begin{array}{ccc}(-1) & \text { id } & (0) \\ k & \longleftarrow & \longleftarrow\end{array}\right) \in \mathbf{C h}(k)$

which allows us to factorize the unique $\mathbf{C h}(k)$-morphism $0 \rightarrow k$ into an acyclic cofibration $0 \rightarrow D$ followed by a fibration $D \rightarrow k$. (Concretely, the latter map is given by id : $k \rightarrow k$ in degree 0 and $0: k \rightarrow 0$ in degree -1 .) Taking the tensor product $(-) \otimes \mathfrak{F}^{*}$ of the factorization $0 \rightarrow D \rightarrow k$ yields a factorization

$$
0 \longrightarrow D \otimes \mathfrak{F}^{*} \stackrel{p}{\longrightarrow} \mathfrak{F}^{*}
$$

of the zero map $0: 0 \rightarrow \mathfrak{F}^{*}$ into a weak equivalence $0 \rightarrow$ $D \otimes \mathfrak{F}^{*}$ followed by a fibration $p: D \otimes \mathfrak{F}^{*} \rightarrow \mathfrak{F}^{*}$. Hence, we have constructed a replacement

$\widetilde{0}:=\operatorname{id} \times p: \widetilde{T}^{*} \mathfrak{F}:=\mathfrak{F} \times\left(D \otimes \mathfrak{F}^{*}\right) \longrightarrow \mathfrak{F} \times \mathfrak{F}^{*}=T^{*} \mathfrak{F}$

of the zero section $0: \mathfrak{F} \rightarrow T^{*} \mathfrak{F}$ by a weakly equivalent fibration.

Let us now compute explicitly the ordinary pullback

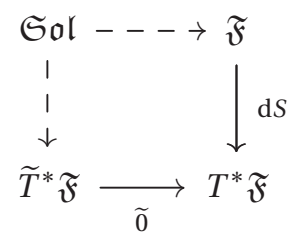


in $\mathbf{C h}(k)$, which provides a model for the desired homotopy pullback (3.26). For this we use that the chain complex $D \otimes \mathfrak{F}^{*}$ is concretely given by

$$
D \otimes \mathfrak{F}^{*}=\left(\Omega^{0}(M) \stackrel{(-2)}{\delta \pi_{1}+\pi_{2}} \Omega^{1}(M) \times \Omega^{(-1)} \Omega^{0}(M)^{(\mathrm{id},-\delta)} \stackrel{(0)}{\leftarrow} \Omega^{1}(M)\right)
$$

and that the $\mathbf{C h}(k)$-morphism $p: D \otimes \mathfrak{F}^{*} \rightarrow \mathfrak{F}^{*}$ reads as

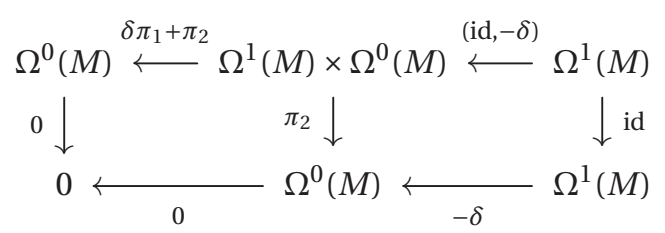

One easily computes the lower horizontal arrow in (3.38) and confirms that the pullback is given by (3.34).

Remark 3.22. The following remarks are in order:

- As a graded vector space, the model $\mathfrak{S o l}$ for the derived critical locus established in Proposition 3.21 agrees with the shifted cotangent bundle over $\mathfrak{F}$. Hence, the above proof of Proposition 3.21 provides a homological explanation for the appearance of shifted cotangent bundles in the calculation of derived critical loci.

- The chain complex Sol in (3.34) has both "stacky" positive degrees and "derived" negative degrees. The different components have a physical interpretation in terms of the BRST/BV formalism: Fields in degree 0 are called gauge fields $A \in \Omega^{1}(M)$ and fields in degree 1 ghost fields $c \in \Omega^{0}(M)$. The fields in negative degrees are called anti fields $A^{\ddagger} \in \Omega^{1}(M)$ and $c^{\ddagger} \in \Omega^{0}(M)$.

Let us now compute the homologies of the chain complex (3.34). In degree 1 we obtain the zeroth de Rham cohomology of the spacetime $M$

$H_{1}(\mathfrak{S o l}) \cong H_{\mathrm{dR}}^{0}(M) \cong \mathbb{R}^{\pi_{0}(M)}$,

where $\pi_{0}(M)$ denotes the set of connected components of $M$. Note that this is always non-zero and it agrees with our computation of $\pi_{1}\left(B G^{\mathrm{con}}(U), A\right)$ for the groupoid of gauge fields in Example 3.2. In degree 0 we obtain the space of gauge equivalences classes of solutions of the Abelian Yang-Mills equation

$H_{0}(\mathfrak{S} \circ \mathfrak{l}) \cong \frac{\left\{A \in \Omega^{1}(M): \delta \mathrm{d} A=0\right\}}{\mathrm{d} \Omega^{0}(M)}$.

Working out the homology in degree -1 is slightly more complicated. Because $M$ is by hypothesis a globally hyperbolic spacetime, one can show that the inhomogeneous Abelian Yang-Mills equation $\delta \mathrm{d} A=j$, for $j \in \Omega^{1}(M)$, has a solution $A \in \Omega^{1}(M)$ if and only if $j$ is $\delta$-exact, i.e. $j=\delta \eta$ for some $\eta \in \Omega^{2}(M)$. (Hint: Apply a gauge transformation to $A$ to fulfill the Lorenz gauge condition $\delta A=0$ and then use standard techniques from the theory of wave equations [3].) Hence,

$\operatorname{Im}\left(\delta \mathrm{d}: \Omega^{1}(M) \rightarrow \Omega^{1}(M)\right)=\delta \Omega^{2}(M)$

is the space of $\delta$-exact 1-forms and the degree -1 homology of (3.34) is

$H_{-1}(\mathfrak{S o l}) \cong H_{\delta}^{1}(M) \cong H_{\mathrm{dR}}^{m-1}(M)$,

where $H_{\delta}^{\bullet}$ is the cohomology of the codifferential. (Recall that $m$ is the dimension of $M$.) Finally, the degree -2 homology of (3.34) is trivial

$H_{-2}(\mathfrak{S o l}) \cong H_{\delta}^{0}(M) \cong H_{\mathrm{dR}}^{m}(M) \cong 0 \quad$,

because the underlying manifold of a globally hyperbolic spacetime is diffeomorphic to $M \cong \mathbb{R} \times \Sigma$, with $\Sigma$ an $m-1$ dimensional manifold. Note that this calculation shows that the $\mathbf{C h}(k)$-morphism

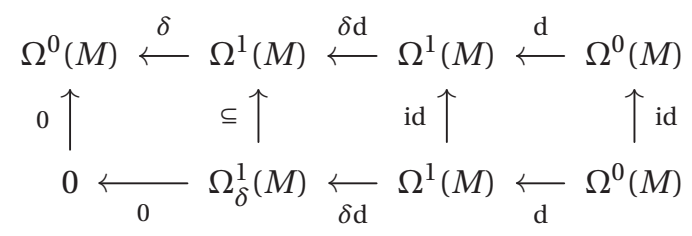

is a quasi-isomorphism, where by $\Omega_{\delta}^{1}(M)$ we denoted the $\delta$-closed 1-forms.

Corollary 3.23. For every globally hyperbolic Lorentzian spacetime $M \in \mathbf{L o c}$, the solution complex $\mathfrak{S o l}$ in (3.34) is weakly equivalent to the smaller chain complex

$\widetilde{S_{o l}}:=\left(\begin{array}{ccc}(-1) & \stackrel{\delta \mathrm{d}}{(0)} \stackrel{(0)}{\leftarrow} \stackrel{(1)}{\leftarrow} \Omega^{1}(M) \stackrel{\stackrel{(1)}{\leftarrow}}{\leftarrow} \Omega^{0}(M)\end{array}\right)$.

We would like to conclude this section with a comment on the time-slice axiom in the present setting. Let us take any Cauchy morphism $f: M \rightarrow N$ in Loc, i.e. $f(M) \subseteq N$ contains a Cauchy surface of $N$. Pullback of differential forms defines a $\mathbf{C h}(k)$-morphism

$f^{*}: \operatorname{Sol}(N) \longrightarrow \operatorname{Sol}(M)$

from the chain complex of solutions on $N$ to the one on $M$. One easily observes that $f^{*}$ is not an isomorphism in $\mathbf{C h}(k)$, but rather a quasi-isomorphism. (The same statements hold true for the smaller complex in (3.47).) Thinking ahead towards homotopical AQFT, this means that one should not expect a strict time-slice axiom to hold true in such gauge theoretic examples, but rather a homotopycoherent generalization of it. 


\section{Homotopy theory of AQFTs}

We develop a general framework for AQFTs with values in the symmetric monoidal model category $\mathbf{C h}(k)$ of chain complexes of $k$-modules. This is motivated by the higher structures arising in gauge theory that we explained in Section 3 above. Concrete examples that fit into our framework are models which are constructed via the perturbative BRST/BV formalism for AQFT, see e.g. [39, 40, 49, 64]. We will assume throughout the whole section that $k \supseteq \mathbb{Q}$ is a commutative unital ring that includes the ring of rational numbers as a subring. (The physically relevant examples are complex numbers $k=\mathbb{C}$ and formal power series $k=\mathbb{C}[[\hbar]]$.) This will considerably simplify our model categorical considerations and arguments. For details on the material presented below we refer to [15].

\subsection{Homotopy theory of algebras over dg-operads}

Colored dg-operads are similar to the Set-valued colored operads from Section 2.2, however with the difference that they have chain complexes of $n$-ary operations. In more detail, a colored dg-operad $\mathscr{O} \in \mathbf{O p}(\mathbf{C h}(k))$ is described by the following data:

- an underlying set of colors;

- for each tuple $(c, t)=\left(\left(c_{1}, \ldots, c_{n}\right), t\right)$ of colors, a chain complex $\mathscr{O}\left(\begin{array}{c}t \\ c\end{array}\right) \in \mathbf{C} \mathbf{h}(k)$ of operations from $\underline{c}$ to $t$;

- composition $\mathbf{C h}(k)$-morphisms $\gamma: \mathscr{O}\left(\begin{array}{c}t \\ \underline{c}\end{array}\right) \otimes \bigotimes_{i=1}^{n} \mathscr{O}\left(\begin{array}{l}c_{i} \\ \underline{b}_{i}\end{array}\right) \rightarrow$ $\left.\mathscr{O}\left(\underline{(}_{1}, \ldots, \underline{b}_{n}\right)\right)$;

- unit $\mathbf{C h}(k)$-morphisms $\mathbb{1}: k \rightarrow \mathscr{O}\left(\begin{array}{l}t \\ t\end{array}\right)$;

- permutation action $\mathbf{C h}(k)$-morphisms $\mathscr{O}(\sigma): \mathscr{O}\left(\begin{array}{c}t \\ c\end{array}\right) \rightarrow$ $\mathscr{O}\left(\begin{array}{c}t \\ c \sigma\end{array}\right)$.

This data has to satisfy the usual associativity, unitality and equivariance conditions, see e.g. [65]. Given any Setvalued colored operad $\mathscr{P} \in \mathbf{O p}$ (Set), one can define a colored dg-operad $\mathscr{P} \otimes k \in \mathbf{O p}(\mathbf{C h}(k))$ by tensoring

$$
\mathscr{P}\left(\begin{array}{l}
t \\
\underline{c}
\end{array}\right) \otimes k:=\bigsqcup_{p \in \mathscr{P}\left(\begin{array}{l}
t \\
\underline{c}
\end{array}\right)} k
$$

each set of operations with the monoidal unit $k \in \mathbf{C h}(k)$.

To every colored dg-operad $\mathscr{O} \in \mathbf{O p}(\mathbf{C h}(k))$ one can assign its category of algebras $\operatorname{Alg}_{\mathscr{C}}(\mathbf{C h}(k))$ with values in the symmetric monoidal model category $\mathbf{C h}(k)$ of chain complexes. Concretely, an $\mathscr{O}$-algebra $A \in \mathbf{A l g}_{\mathscr{O}}(\mathbf{C h}(k))$ is a collection of chain complexes $A_{c} \in \mathbf{C h}(k)$, for all colors $c$, together with $\mathbf{C h}(k)$-morphisms

$$
A: \mathscr{O}\left(\begin{array}{c}
t \\
\underline{c}
\end{array}\right) \otimes \bigotimes_{i=1}^{n} A_{c_{i}} \longrightarrow A_{t}
$$

that encode the actions of the chain complexes of operations. Of course, various compatibility conditions with the operad structure on $\mathscr{O}$ must be fulfilled, see e.g. [65].

Recalling that $\mathbf{C h}(k)$ is a (symmetric monoidal) model category, with weak equivalences the quasi-isomorphisms, it is natural to ask whether $\operatorname{Alg}_{\mathscr{O}}(\mathbf{C h}(k))$ is a model category too. In general, this turns out to be a complicated question and there is a large amount of literature on model structures for operad algebras in model categories, see e.g. $[17,18,25,45,46,59,63]$. The case of relevance to us has been understood by Hinich $[45,46]$, who has proven the following result.

Theorem 4.1. Let $\mathscr{O} \in \mathbf{O p}(\mathbf{C h}(k))$ be any colored $d g$ operad. Define a morphism $\kappa: A \rightarrow B$ in $\mathbf{A l g}_{\mathscr{O}}(\mathbf{C h}(k))$ to be

- a weak equivalence if each component $\kappa_{c}: A_{c} \rightarrow B_{c}$ is a weak equivalence in $\mathbf{C h}(k)$, i.e. a quasi-isomorphism;

- a fibration if each component $\kappa_{c}: A_{c} \rightarrow B_{c}$ is a fibration in $\mathbf{C h}(k)$, i.e. degree-wise surjective;

- a cofibration if it has the left lifting property with respect to all acyclic fibrations.

If $k \supseteq \mathbb{Q}$, then these choices endow $\operatorname{Alg}_{\mathscr{O}}(\mathbf{C h}(k))$ with the structure of a model category. In this model structure every object $A \in \mathbf{A l g}_{\mathscr{O}}(\mathbf{C h}(k))$ is fibrant.

Let us now consider any $\mathbf{O p}(\mathbf{C h}(k))$-morphism $\phi: \mathscr{O} \rightarrow$ $\mathscr{P}$ between two colored dg-operads. There is an associated pullback functor $\phi^{*}: \mathbf{A l g}_{\mathscr{P}}(\mathbf{C h}(k)) \rightarrow \operatorname{Alg}_{\mathscr{O}}(\mathbf{C h}(k))$ between the categories of algebras, which admits a left adjoint given by operadic left Kan extension, i.e. we obtain an adjunction

$$
\phi_{!}: \operatorname{Alg}_{\mathscr{O}}(\mathbf{C h}(k)) \rightleftarrows \operatorname{Alg}_{\mathscr{P}}(\mathbf{C h}(k)): \phi^{*} \quad
$$

It is easy to see that this adjunction is compatible with the model structures in Theorem 4.1.

Proposition 4.2. For every $\mathrm{Op}(\mathbf{C h}(k))$-morphism $\phi: \mathscr{O} \rightarrow$ $\mathscr{P}$, the adjunction (4.3) is a Quillen adjunction. Moreover, the right adjoint $\phi^{*}$ preserves weak equivalences.

Remark 4.3. In general, the left adjoint functor $\phi_{!}$in (4.3) does not preserve weak equivalences and has to be derived. Choosing any natural cofibrant replacement $\left(Q: \operatorname{Alg}_{\mathscr{O}}(\mathbf{C h}(k)) \rightarrow \operatorname{Alg}_{\mathscr{O}}(\mathbf{C h}(k)), q: Q \stackrel{\sim}{\rightarrow} \mathrm{id}\right)$, we define as usual the left derived functor by

$\mathbb{L} \phi_{!}:=\phi_{!} Q: \operatorname{Alg}_{\mathscr{O}}(\mathbf{C h}(k)) \longrightarrow \operatorname{Alg}_{\mathscr{P}}(\mathbf{C h}(k))$.

By construction, $\mathbb{L} \phi$ ! does preserve weak equivalences. Because by Theorem 4.1 each object in $\operatorname{Alg}_{\mathscr{P}}(\mathbf{C h}(k))$ is fibrant, we will always choose in what follows the trivial fibrant replacement $\left(R=\mathrm{id}: \operatorname{Alg}_{\mathscr{P}}(\mathbf{C h}(k)) \rightarrow\right.$ 
$\left.\operatorname{Alg}_{\mathscr{P}}(\mathbf{C h}(k)), r=\mathrm{id}: \mathrm{id} \stackrel{\sim}{\rightarrow} R\right)$, which means that the right derived functor

$\mathbb{R} \phi^{*}:=\phi^{*} R=\phi^{*}: \operatorname{Alg}_{\mathscr{P}}(\mathbf{C h}(k)) \longrightarrow \operatorname{Alg}_{\mathscr{O}}(\mathbf{C h}(k))$

agrees with the underived functor.

Next, we shall briefly discuss the concept of $\Sigma$-cofibrant resolutions of colored dg-operads and the associated concept of homotopy algebras. In what follows we fix a nonempty set $\mathfrak{C} \in$ Set of colors and consider the subcategory $\mathbf{O p}_{\mathfrak{C}}(\mathbf{C h}(k)) \subseteq \mathbf{O p}(\mathbf{C h}(k))$ of colored dg-operads with fixed set of colors $\mathfrak{C}$ and morphisms acting as the identity on colors. Under our hypothesis $k \supseteq \mathbb{Q}$, Hinich's results $[45,46]$ imply that $\mathbf{O} \mathbf{p}_{\mathfrak{C}^{\mathfrak{c}}}(\mathbf{C h}(k))$ is a model category with weak equivalences and fibrations defined component-wise and cofibrations defined by the left lifting property. Let us introduce the following standard terminology.

Definition 4.4. A $\mathfrak{C}$-colored dg-operad $\mathscr{O} \in \mathbf{O} \mathbf{p}_{\mathfrak{C}}(\mathbf{C h}(k))$ is called $\Sigma$-cofibrant if each component $\mathscr{O}\left(\begin{array}{l}t \\ \underline{c}\end{array}\right)$ is a cofibrant object in the projective model structure on the functor category $\mathbf{C h}(k)^{\Sigma_{\underline{c}}}$, where $\Sigma_{\underline{c}} \subseteq \Sigma_{n}$ is the stabilizer subgroup of the tuple $\underline{c}=\left(c_{1}, \ldots, c_{n}\right)$ of colors.

Cofibrant dg-operads, i.e. cofibrant objects in the model category $\mathbf{O p}_{\mathfrak{C}}(\mathbf{C h}(k))$, are in particular $\Sigma$-cofibrant, cf. [17, Proposition 4.3]. However, the converse is not true since e.g. the commutative dg-operad Com is $\Sigma$ cofibrant but not cofibrant. The relevance of $\Sigma$-cofibrant dg-operads is that their categories of algebras behave well with respect to weak equivalences. More precisely, the relevant result $[45,46]$ is as follows.

Theorem 4.5. Let $\phi: \mathscr{O} \rightarrow \mathscr{P}$ be a weak equivalence between $\Sigma$-cofibrant colored dg-operads $\mathscr{O}, \mathscr{P} \in \mathbf{O p}_{\mathfrak{C}}(\mathbf{C h}(k))$. Then the corresponding Quillen adjunction (4.3) is a Quillen equivalence.

With this preparation we can now finally define the concept of homotopy algebras over colored dg-operads.

Definition 4.6. Let $\mathscr{O} \in \mathbf{O p}_{\mathfrak{C}}(\mathbf{C h}(k))$ be a colored dgoperad.

1. A $\Sigma$-cofibrant resolution of $\mathscr{O}$ is a $\Sigma$-cofibrant colored dg-operad $\mathscr{O}_{\infty} \in \mathbf{O} \mathbf{p}_{\mathfrak{C}}(\mathbf{C h}(k))$ together with an acyclic fibration $w: \mathscr{O}_{\infty} \rightarrow \mathscr{O}$ in $\mathbf{O p}_{\mathfrak{C}}(\mathbf{C h}(k))$.

2. The model category of homotopy $\mathscr{O}$-algebras is the model category $\operatorname{Alg}_{\mathscr{O}_{\infty}}(\mathbf{C h}(k))$ of algebras over a $\Sigma$ cofibrant resolution $w: \mathscr{O}_{\infty} \rightarrow \mathscr{O}$.

Remark 4.7. It is natural to ask whether the concept of homotopy $\mathscr{O}$-algebras depends on the chosen resolution. Given two $\Sigma$-cofibrant resolutions $w: \mathscr{O}_{\infty} \rightarrow \mathscr{O}$ and $w^{\prime}: \mathscr{O}_{\infty}^{\prime} \rightarrow \mathscr{O}$, and taking also a cofibrant replacement $q: Q \mathscr{O} \rightarrow \mathscr{O}$, we obtain a commutative diagram

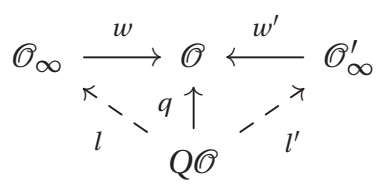

in $\mathbf{O} \mathbf{p}_{\mathfrak{C}}(\mathbf{C h}(k))$. The dashed arrows exist due to the left lifting property, because $Q \mathscr{O}$ is by construction a cofibrant dg-operad and $w, w^{\prime}$ are acyclic fibrations. By Proposition 4.2 we obtain a zig-zag

$$
\operatorname{Alg}_{\mathscr{O}_{\infty}}(\mathbf{C h}(k)) \underset{l^{*}}{\stackrel{l_{!}}{\leftrightarrows}} \operatorname{Alg}_{Q \mathscr{Q}}(\mathbf{C h}(k)) \underset{l^{* *}}{\stackrel{l_{!}^{\prime}}{\rightleftarrows}} \operatorname{Alg}_{\mathscr{O}_{\infty}^{\prime}}(\mathbf{C h}(k))
$$

of Quillen adjunctions. Because $\mathscr{O}_{\infty}, \mathscr{O}_{\infty}^{\prime}$ and also the cofibrant dg-operad $Q \mathscr{O}$ are $\Sigma$-cofibrant, Theorem 4.5 implies that this is a zig-zag of Quillen equivalences and hence that the model categories $\mathbf{A l g}_{\mathscr{C}_{\infty}}(\mathbf{C h}(k))$ and $\mathbf{A l g}_{\mathscr{O}_{\infty}^{\prime}}(\mathbf{C h}(k))$ of homotopy $\mathscr{O}$-algebras are equivalent in this sense.

Example 4.8. The following are standard examples of homotopy algebras:

- $A_{\infty}$-algebras are homotopy algebras over the associative operad As;

- $E_{\infty}$-algebras are homotopy algebras over the commutative operad Com;

- $L_{\infty}$-algebras are homotopy algebras over the Lie operad Lie;

- homotopy-coherent diagrams are homotopy algebras over the diagram operad Diag $_{\mathbf{C}}$.

\subsection{AQFT model categories and Quillen adjunctions}

Let $\overline{\mathbf{C}}=(\mathbf{C}, \perp)$ be any orthogonal category and $\mathscr{O}_{\overline{\mathbf{C}}} \epsilon$ $\mathbf{O p}$ (Set) the corresponding AQFT operad from Theorem 2.8. The following result is fundamental for the developments throughout the whole section.

Theorem 4.9. For every orthogonal category $\overline{\mathbf{C}}$, the category QFT $(\overline{\mathbf{C}})$ of $\mathbf{C h}(k)$-valued AQFTs on $\overline{\mathbf{C}}$ (cf. Definition 2.3) is a model category with respect to the following choices: A morphism $\zeta: \mathfrak{A} \rightarrow \mathfrak{B}$ in $\mathbf{Q F T}(\overline{\mathbf{C}})$ (i.e. a natural transformation between the underlying functors $\left.\mathbf{C} \rightarrow \operatorname{Alg}_{\mathrm{As}}(\mathbf{C h}(k))\right)$ is

- a weak equivalence if each component $\zeta_{c}: \mathfrak{A}(c) \rightarrow \mathfrak{B}(c)$ is a quasi-isomorphism;

- a fibration if each component $\zeta_{c}: \mathfrak{A}(c) \rightarrow \mathfrak{B}(c)$ is degreewise surjective;

- a cofibration if it has the left lifting property with respect to all acyclic fibrations. 
Proof. This is a direct consequence of Theorem 4.1 and the chain of isomorphism $\mathbf{Q F T}(\overline{\mathbf{C}}) \cong \operatorname{Alg}_{\mathscr{O}_{\overline{\mathrm{C}}}}(\mathbf{C h}(k)) \cong$ $\operatorname{Alg}_{\mathscr{O}_{\overline{\mathrm{C}}} \otimes k}(\mathbf{C h}(k))$. The first step is Theorem 2.9 and for the second step one easily checks that the category $\operatorname{Alg}_{\mathscr{O}_{\overline{\mathrm{C}}}}(\mathbf{C h}(k))$ of $\mathbf{C h}(k)$-valued algebras over the Set-valued AQFT operad $\mathscr{O}_{\overline{\mathbf{C}}} \in \mathbf{O p}$ (Set) is (isomorphic to) the category $\operatorname{Alg}_{\mathscr{C}_{\overline{\mathbf{C}} \otimes k}}(\mathbf{C h}(k))$ of algebras over the corresponding AQFT dg-operad $\mathscr{O}_{\overline{\mathbf{C}}} \otimes k \in \mathbf{O p}(\mathbf{C h}(k))$.

Remark 4.10. Our rigorous concept of weak equivalences for $\mathbf{C h}(k)$-valued AQFTs established in Theorem 4.9 agrees with the less formal notions used in concrete applications of the BRST/BV formalism, cf. [39, 40, 49]. In particular, the usual technique of "adding auxiliary fields without changing homologies" can be understood rigorously from our model categorical perspective.

In the context of $\mathbf{C h}(k)$-valued AQFTs, the universal constructions from Section 2.3 have to be derived in order to be consistent with the concept of weak equivalences introduced in Theorem 4.9. Recall from Theorem 2.12 that every orthogonal functor $F: \overline{\mathbf{C}} \rightarrow \overline{\mathbf{D}}$ defines an adjunction

$$
F_{!}: \mathbf{Q F T}(\overline{\mathbf{C}}) \rightleftarrows \mathbf{Q F T}(\overline{\mathbf{D}}): F^{*}
$$

between the corresponding categories of $\mathbf{C h}(k)$-valued AQFTs. As a direct consequence of Proposition 4.2, we obtain the following result.

Proposition 4.11. For every orthogonal functor $F: \overline{\mathbf{C}} \rightarrow \overline{\mathbf{D}}$, the adjunction (4.8) is a Quillen adjunction. Moreover, the right adjoint $F^{*}$ preserves weak equivalences.

As explained in Remark 4.3, the left adjoint functor $F_{\text {! }}$ has to be derived, e.g. by choosing a natural cofibrant replacement. This has consequences for the examples of universal constructions discussed in Section 2.3.

Example 4.12. Let $j: \overline{\mathbf{C}} \rightarrow \overline{\mathbf{D}}$ be a full orthogonal subcategory embedding and consider as in (2.18) the extension/restriction adjunction $j ! \dashv j^{*}$ for $\mathbf{C h}(k)$-valued AQFTs. By Proposition 4.11, this is a Quillen adjunction and hence we can construct the left derived extension functor $\mathbb{L} j !:=j ! Q: \mathbf{Q F T}(\overline{\mathbf{C}}) \rightarrow \mathbf{Q F T}(\overline{\mathbf{D}})$. We would like to emphasize again that it is the derived functor $\mathbb{L} j$ ! that defines a meaningful local-to-global extension for $\mathbf{C h}(k)$ valued AQFTs and not the underived functor $j_{\text {! }}$, because the latter in general does not preserve weak equivalences. (See [15, Appendix A] for concrete examples.) This in particular means that our definition of descent via $j$-locality (cf. Definition 2.17) has to be adapted in order to be homotopically meaningful. Following [20], we say that
$\mathfrak{A} \in \mathbf{Q F T}(\overline{\mathbf{D}})$ is homotopy $j$-local if the corresponding component

$$
\mathbb{L} j ! j^{*} \mathfrak{A}=j ! Q j^{*} \mathfrak{A} \stackrel{j_{!} q_{j^{*} \mathfrak{A}}}{\longrightarrow} j ! j^{*} \mathfrak{A} \stackrel{\epsilon_{\mathfrak{A}}}{\longrightarrow} \mathfrak{A}
$$

of the derived counit is a weak equivalence. It is easy to prove that the derived extension $\mathbb{L} j ! \mathfrak{B}$ of every $\mathfrak{B} \in$ QFT $(\overline{\mathbf{C}})$ is homotopy $j$-local. Toy-models of homotopy $j$-local AQFTs that are inspired by gauge theory are presented in Section 4.4 below.

Example 4.13. Let $L: \overline{\mathbf{C}} \rightarrow \overline{\mathbf{C}\left[W^{-1}\right]}$ be an orthogonal localization and consider as in (2.19) the time-slicification adjunction $L ! \dashv L^{*}$ for $\mathbf{C h}(k)$-valued AQFTs. By Proposition 4.11, this is a Quillen adjunction and hence we can construct the left derived time-slicification functor $\mathbb{L} L_{!}:=L_{!} Q: \mathbf{Q F T}(\overline{\mathbf{C}}) \rightarrow \mathbf{Q F T}\left(\overline{\mathbf{C}\left[W^{-1}\right]}\right)$. Our concept of $W$ constancy from Corollary 2.22 has to be adapted in order to be homotopically meaningful. Following [20], we say that $\mathfrak{A} \in \mathbf{Q F T}(\overline{\mathbf{C}})$ is homotopy $W$-constant if the corresponding component

$$
Q \mathfrak{A} \stackrel{\eta_{Q \mathfrak{A}}}{\longrightarrow} L^{*} L ! Q \mathfrak{A}=L^{*} \llbracket L ! \mathfrak{A}
$$

of the derived unit is a weak equivalence. Note that homotopy $W$-constancy can be interpreted as a homotopy theoretic generalization of the time-slice axiom. We expect that this will be useful for formalizing the weaker concept of time-slice axiom appearing in derived geometry, see the end of Section 3.4.

Open Problem 4.14. The formal properties of the derived unit and counit of the time-slicification adjunction $L_{!} \dashv L^{*}$ are harder to understand than the ones for the extension/restriction adjunction $j$ ! $\dashv j^{*}$. In particular, even though the underived counit $\epsilon$ is a natural isomorphism by Proposition 2.21, it is unclear if the derived counit

$\mathbb{L} L_{!} L^{*}=L_{!} Q L^{*} \stackrel{L_{!} q L^{*}}{\longrightarrow} L_{!} L^{*} \stackrel{\epsilon}{\longrightarrow}$ id

is a natural weak equivalence. As a consequence, it is currently unclear to us if theories of the form $L^{*} \mathfrak{B}$, for $\mathfrak{B} \in \mathbf{Q F T}\left(\overline{\mathbf{C}\left[W^{-1}\right]}\right)$, are homotopy $W$-constant.

\subsection{Homotopy-coherent AQFTs}

The aim of this section is to study homotopy algebras over the AQFT dg-operad $\mathscr{O}_{\overline{\mathbf{C}}} \otimes k \in \mathbf{O p}(\mathbf{C h}(k))$, which we shall also call homotopy AQFTs. The following fundamental theorem is proven in [15]. Recall from Definitions 4.4 and 4.6 
the concepts of $\Sigma$-cofibrant dg-operads and $\Sigma$-cofibrant resolutions.

Theorem 4.15. Let us assume as before that $k \supseteq \mathbb{Q}$ includes the rationals. For every orthogonal category $\overline{\mathbf{C}}$, the $A Q F T$ dg-operad $\mathscr{O}_{\overline{\mathbf{C}}} \otimes k \in \mathbf{O} \mathbf{p}(\mathbf{C h}(k))$ is $\Sigma$-cofibrant. As a consequence of Theorem 4.5, every $\Sigma$-cofibrant resolution $w: \mathscr{O}_{\infty} \rightarrow \mathscr{O}_{\overline{\mathbf{C}}} \otimes k$ induces a Quillen equivalence

$$
w_{!}: \mathbf{A l g}_{\mathscr{O}_{\infty}}(\mathbf{C h}(k)) \rightleftarrows \mathbf{Q F T}(\overline{\mathbf{C}}): w^{*}
$$

Remark 4.16. This result can be interpreted as a strictification theorem for homotopy AQFTs. Indeed, given any homotopy AQFT $\mathfrak{A}_{\infty} \in \mathbf{A l g}_{\mathscr{O}_{\infty}}(\mathbf{C h}(k))$, a cofibrant replacement and the derived unit of $w_{!} \dashv w^{*}$ defines a zig-zag

$$
\mathfrak{A}_{\infty} \stackrel{q_{\mathfrak{A}_{\infty}}}{\longleftarrow} Q \mathfrak{A}_{\infty} \stackrel{\eta_{Q \mathfrak{A}_{\infty}}}{\longrightarrow} w^{*} \mathbb{L} w ! \mathfrak{A}_{\infty}
$$

of weak equivalences between $\mathfrak{A}_{\infty}$ and the strict AQFT $w^{*} \llbracket w_{!} \mathfrak{A}_{\infty}$.

So does this mean that homotopy AQFTs are not interesting and important at all? The answer to this question is clearly no, because certain interesting constructions naturally define non-strict homotopy AQFTs. For instance, let us recall from Example 3.18 that taking derived smooth normalized cochain algebras on a functor $\mathfrak{F}: \mathbf{C}^{\text {op }} \rightarrow \mathbf{S t}_{\infty} \subseteq$ $\mathbf{H}_{\infty}$ that assigns $\infty$-stacks of gauge fields to spacetimes defines a functor $\mathfrak{A}:=\mathbb{L} N^{\infty *}(\mathfrak{F}(-), k): \mathbf{C} \rightarrow \operatorname{Alg}_{\mathscr{E}_{\infty}}(\mathbf{C h}(k))$ with values in $E_{\infty}$-algebras. We shall show in Example 4.20 below that this can be interpreted as a non-strict homotopy AQFT. A further class of examples is given in Section 4.5. Note that even though each of these non-strict homotopy AQFTs can be strictified by Theorem 4.15, such strictifications are hard to describe explicitly and thus it is often useful in practice to work directly with the weaker model.

There exist of course many different $\Sigma$-cofibrant resolutions of the AQFT dg-operad $\mathscr{O}_{\overline{\mathbf{C}}} \otimes k \in \mathbf{O p}(\mathbf{C h}(k))$, which describe homotopy AQFTs whose algebraic structures (functoriality, associativity, $\perp$-commutativity, etc.) are weakened in a homotopy-coherent sense by a certain extent. The strictest possible resolution is given by the identity id : $\mathscr{O}_{\overline{\mathbf{C}}} \otimes k \rightarrow \mathscr{O}_{\overline{\mathbf{C}}} \otimes k$ and homotopy AQFTs with respect to this resolution are precisely strict AQFTs. A very weak resolution, called the Boardman-Vogt resolution, has been studied for our AQFT operads by Yau in [66]. The resulting homotopy AQFTs are, roughly speaking, homotopy-coherent diagrams of $A_{\infty}$-algebra that satisfy a homotopy-coherent $\perp$-commutativity property. Motivated by our examples from Example 3.18 and Section
4.5, we shall study below a particular $\Sigma$-cofibrant resolution $w: \mathscr{O}_{\overline{\mathbf{C}}} \otimes \mathscr{E}_{\infty} \rightarrow \mathscr{O}_{\overline{\mathbf{C}}} \otimes k$ of the AQFT dg-operad that is obtained by a component-wise tensoring with the BarrattEccles $E_{\infty}$-operad $\mathscr{E}_{\infty} \in \mathbf{O p}(\mathbf{C h}(k))$. This describes homotopy AQFTs that are strictly functorial, however with a homotopy-coherent $\perp$-commutativity property.

Without going into any details, let us recall from [16] that the Barratt-Eccles dg-operad $\mathscr{E}_{\infty} \in \mathbf{O p}(\mathbf{C h}(k))$ is a $\Sigma$ cofibrant resolution $w: \mathscr{E}_{\infty} \rightarrow$ Com of the commutative dg-operad. The usual $\mathbf{O p}(\mathbf{C h}(k))$-morphism As $\rightarrow$ Com from the associative to the commutative dg-operad factors through $\mathscr{E}_{\infty}$, i.e. we have a chain of operad maps

$$
\text { As } \stackrel{i}{\longrightarrow} \mathscr{E}_{\infty} \stackrel{w}{\longrightarrow} \text { Com }
$$

The induced Quillen adjunctions imply that each commutative dg-algebra $C \in \mathbf{A l g}_{\text {Com }}(\mathbf{C h}(k))$ can be interpreted as a strictly commutative $E_{\infty}$-algebra $w^{*} C \in \operatorname{Alg}_{\mathscr{E}_{\infty}}(\mathbf{C h}(k))$. Moreover, each $E_{\infty}$-algebra $A \in \operatorname{Alg}_{\mathscr{E}_{\infty}}(\mathbf{C h}(k))$ has an underlying dg-algebra $i^{*} A \in \operatorname{Alg}_{\mathrm{As}}(\mathbf{C h}(k))$, which is in general noncommutative unless the $E_{\infty}$-algebra is strictly commutative.

For every orthogonal category $\overline{\mathbf{C}}$, we define a colored dg-operad $\mathscr{O}_{\overline{\mathbf{C}}} \otimes \mathscr{E}_{\infty} \in \mathbf{O p}(\mathbf{C h}(k))$ by the component-wise tensoring

$\left(\mathscr{O}_{\overline{\mathbf{C}}} \otimes \mathscr{E}_{\infty}\right)\left(\begin{array}{l}t \\ c\end{array}\right):=\mathscr{O}_{\overline{\mathbf{C}}}\left(\begin{array}{l}t \\ \underline{c}\end{array}\right) \otimes \mathscr{E}_{\infty}(n) \in \mathbf{C h}(k)$

of the AQFT operad with the Barratt-Eccles $E_{\infty}$-operad, where $n$ is the length of the tuple of colors $\underline{c}$. The following result was proven in [15].

Theorem 4.17. Let us assume as before that $k \supseteq \mathbb{Q}$ includes the rationals. For every orthogonal category $\overline{\mathbf{C}}$, the $\mathbf{O p}(\mathbf{C h}(k))$-morphism

$w_{\overline{\mathbf{C}}}:=\mathrm{id} \otimes w: \mathscr{O}_{\overline{\mathbf{C}}} \otimes \mathscr{E}_{\infty} \longrightarrow \mathscr{O}_{\overline{\mathbf{C}}} \otimes \mathrm{Com} \cong \mathscr{O}_{\overline{\mathbf{C}}} \otimes k$

defines a $\Sigma$-cofibrant resolution of the AQFT dg-operad. These resolutions are natural in the orthogonal category $\overline{\mathbf{C}} \in$ OrthCat.

Definition 4.18. Let $\overline{\mathbf{C}}$ be an orthogonal category. We denote by

$\mathbf{Q F T}_{\infty}(\overline{\mathbf{C}}):=\operatorname{Alg}_{\mathscr{C}_{\overline{\mathbf{C}}}^{\otimes \mathscr{E}_{\infty}}}(\mathbf{C h}(k))$

the model category of homotopy AQFTs on $\overline{\mathbf{C}}$ that correspond to the $\Sigma$-cofibrant resolution from Theorem 4.17.

Remark 4.19. Our universal constructions for strict $\mathbf{C h}(k)$-valued AQFTs from Section 4.2 immediately generalize to the case of homotopy AQFTs. In particular, for 
every orthogonal functor $F: \overline{\mathbf{C}} \rightarrow \overline{\mathbf{D}}$ one obtains a Quillen adjunction

$$
F_{!}: \mathbf{Q F T}_{\infty}(\overline{\mathbf{C}}) \rightleftarrows \mathbf{Q F T}_{\infty}(\overline{\mathbf{D}}): F^{*}
$$

between the corresponding model categories of homotopy AQFTs. Interesting examples are again extension/restriction adjunctions induced by full orthogonal subcategory embeddings or time-slicification adjunctions induced by orthogonal localizations.

Example 4.20. Given any small category $\mathbf{C}$, we can choose the maximal orthogonality relation

$\perp^{\max }:=\operatorname{Mor}_{\mathrm{t}} \times{ }_{\mathrm{t}}$ MorC

and define an orthogonal category $\overline{\mathbf{C}}^{\max }:=\left(\mathbf{C}, \perp{ }^{\max }\right)$. One easily checks that the category

$\mathbf{Q F T}\left(\overline{\mathbf{C}}^{\mathrm{max}}\right) \cong \operatorname{Alg}_{\mathrm{Com}}(\mathbf{C h}(k)) \mathbf{C}$

of strict AQFTs on $\overline{\mathbf{C}}^{\text {max }}$ is the category of functors from $\mathbf{C}$ to commutative dg-algebras and that the category

$\mathbf{Q F T}_{\infty}\left(\overline{\mathbf{C}}^{\mathrm{max}}\right) \cong \operatorname{Alg}_{\mathscr{E}_{\infty}}(\mathbf{C h}(k))^{\mathbf{C}}$

of homotopy AQFTs (in the sense of Definition 4.18) on $\overline{\mathbf{C}}^{\text {max }}$ is the category of functors from $\mathbf{C}$ to $E_{\infty}$-algebras. In particular, the derived smooth normalized cochain algebras on diagrams of $\infty$-stacks from Example 3.18 define examples of such homotopy AQFTs.

\subsection{Derived local-to-global constructions}

In this section we shall present concrete results on derived local-to-global extensions in a simplified setting. In particular, we shall show that certain simplified toy-models for topological AQFTs satisfy a homotopy $j$-locality property in the sense of Example 4.12. Physically, these results should be interpreted as a homotopical descent condition for such AQFTs. For the technical details we refer to [15].

Let Man be the category of oriented $m$-dimensional manifolds of finite type with morphisms given by orientation preserving open embeddings. We endow Man with the maximal orthogonality relation from Example 4.20 , which defines an orthogonal category $\overline{\operatorname{Man}}^{\max }:=$ (Man, $\perp{ }^{\text {max }}$ ). Let further $\overline{\text { Disk }}^{\text {max }} \subseteq \overline{\text { Man }}^{\text {max }}$ be the full orthogonal subcategory of all manifolds diffeomorphic to $\mathbb{R}^{m}$ and denote the corresponding full orthogonal subcategory embedding by $j: \overline{\text { Disk }}^{\text {max }} \rightarrow \overline{\text { Man }}^{\text {max }}$. We are interested in describing the left derived functor $\mathbb{L} j$ ! of the associated Quillen adjunction

$$
j_{!}: \mathbf{Q F T}_{\infty}\left(\overline{\text { Disk }}^{\max }\right) \rightleftarrows \mathbf{Q F T}_{\infty}\left(\overline{\mathbf{M a n}}^{\max }\right): j^{*} .
$$

The following technical theorem is the key ingredient for our computations. Its proof uses Lurie's Seifert-van Kampen theorem [56, Appendix A.3.1] and is presented in [15].

Theorem 4.21. Suppose that $\mathfrak{A} \in \mathbf{Q F T}_{\infty}\left(\overline{\text { Disk }}^{\text {max }}\right)$ is weakly equivalent to a constant functor Disk $\rightarrow \operatorname{Alg}_{\mathscr{E}_{\infty}}(\mathbf{C h}(k))$ whose value we denote by $A \in \mathbf{A l g}_{\mathscr{E}_{\infty}}(\mathbf{C h}(k))$. Then the derived extension $\mathbb{L} ! \mathfrak{A} \in \mathbf{Q F T}_{\infty}\left(\overline{\mathbf{M a n}}^{\max }\right)$ may be computed object-wise for each $M \in \operatorname{Man}$ by

$(\mathbb{L} j ! \mathfrak{A})(M)=\operatorname{Sing}(M)^{\mathbb{Q}} \otimes \in \mathbf{A l g}_{\mathscr{E}_{\infty}}(\mathbf{C h}(k))$,

where $\operatorname{Sing}(M) \in \mathbf{s S e}$ is the simplicial set of singular simplices in $M$ and $\stackrel{\mathbb{Q}}{\otimes}$ is the derived sSet-tensoring for $E_{\infty}$ algebras, cf. [41, 43].

Remark 4.22. In [43] the $E_{\infty}$-algebra $\operatorname{Sing}(M) \stackrel{\mathbb{Q}}{\otimes} A$ is also referred to as the derived higher Hochschild chains on $\operatorname{Sing}(M)$ with coefficients in $A$.

Example 4.23. Our first example is inspired by DijkgraafWitten theory. Let us consider a gauge theory whose fields on $M$ are described by the groupoid $\mathfrak{B u n} \mathfrak{n}_{G}(M) \in$ Grpd of principal $G$-bundles on $M$ for a finite nilpotent group $G$. By [62, Lemma 2.8], the nerve $B \mathfrak{B u n} \mathfrak{u}_{G}(M) \in$ sSet is weakly equivalent to the simplicial mapping space $B G^{\operatorname{Sing}(M)} \in$ sSet, where $B G \in \mathbf{s S e t}$ is the nerve of the groupoid $* / / G$. We define a homotopy AQFT $\mathfrak{A} \in \mathbf{Q F T}_{\infty}\left(\overline{\mathbf{M a n}}^{\text {max }}\right)$ by forming on each $M \in$ Man the normalized cochain algebra

$\mathfrak{A}(M):=N^{*}\left(B G^{\operatorname{Sing}(M)}, k\right) \in \mathbf{A l g}_{\mathscr{E}_{\infty}}(\mathbf{C h}(k))$.

The restriction $j^{*} \mathfrak{A} \in \mathbf{Q F T}_{\infty}\left(\overline{\text { Disk }}^{\text {max }}\right)$ to disks is weakly equivalent to a constant functor with value $N^{*}(B G, k)$ because $\operatorname{Sing}(U) \rightarrow *$ is a weak equivalence for every $U \in$ Disk. Hence, we can apply Theorem 4.21 and compute

$$
\begin{aligned}
\left(\mathbb{L} j ! j^{*} \mathfrak{A}\right)(M) & \simeq \operatorname{Sing}(M)^{\mathbb{Q}} N^{*}(B G, k) \\
& \simeq N^{*}\left(B G^{\operatorname{Sing}(M)}, k\right)=\mathfrak{A}(M),
\end{aligned}
$$

for all $M \in$ Man. The second step follows from [2, Proposition 5.3] and it uses that $G$ is a finite nilpotent group. Summing up, we have seen that the present toy-model of a homotopy AQFT $\mathfrak{A} \in \mathbf{Q F T}_{\infty}\left(\overline{\mathbf{M a n}}^{\text {max }}\right)$ on $\overline{\mathbf{M a n}}^{\text {max }}$ is weakly equivalent to the derived extension $\mathbb{L} j$ ! of its restriction to disks. As a consequence, it is also homotopy $j$-local in the sense of Example 4.12, which one should interpret as a homotopical descent condition. 
Example 4.24. Our second example is inspired by linear Chern-Simons theory with structure group $\mathbb{R}$, i.e. flat principal $\mathbb{R}$-bundles with connections on 2 -dimensional surfaces. In what follows Man will denote the category of 2-dimensional oriented manifolds and we take $k=\mathbb{R}$. From the perspective of derived geometry of linear gauge fields (cf. Section 3.4), the linear classical observables for this theory on $M \in$ Man are described by the (-1)-shifted compactly supported de Rham complex

$\Omega_{c}^{\bullet}(M)[-1]:=\left(\Omega_{c}^{(-1)}(M) \stackrel{\mathrm{d}}{\leftarrow} \Omega_{c}^{(0)} \stackrel{(\mathrm{d}}{\leftarrow} \Omega_{c}^{(1)}(M)\right)$

We define a homotopy AQFT $\mathfrak{A} \in \mathbf{Q F T}_{\infty}\left(\overline{\mathbf{M a n}}^{\text {max }}\right)$ by forming on each $M \in$ Man the free $E_{\infty}$-algebra

$\mathfrak{A}(M):=\mathbb{E}_{\infty}\left(\Omega_{c}^{\bullet}(M)[-1]\right) \in \operatorname{Alg}_{\mathscr{E}_{\infty}}(\mathbf{C h}(k))$

over this complex. One should interpret this as classical polynomial observables for linear Chern-Simons theory. The restriction $j^{*} \mathfrak{A} \in \mathbf{Q F T}_{\infty}\left(\overline{\text { Disk }}^{\text {max }}\right)$ to disks is weakly equivalent to a constant functor with value $\mathbb{E}_{\infty}(\mathbb{R}[1])$ because the integration map $\int_{U}: \Omega_{c}^{\bullet}(U)[-1] \rightarrow \mathbb{R}[1]$ is a weak equivalence for every $U \in$ Disk. Hence, we can apply Theorem 4.21 and compute

$$
\begin{aligned}
& \left(\mathbb{L} j ! j^{*} \mathfrak{A}\right)(M) \simeq \operatorname{Sing}(M) \stackrel{\mathbb{Q}}{\otimes} \mathbb{E}_{\infty}(\mathbb{R}[1]) \\
& \simeq \operatorname{Sing}(M) \otimes \mathbb{E}_{\infty}(\mathbb{R}[1]) \\
& \simeq \mathbb{E}_{\infty}\left(N_{*}(\operatorname{Sing}(M), \mathbb{R}) \otimes \mathbb{R}[1]\right),
\end{aligned}
$$

for all $M \in$ Man. In the second step we used that for free $E_{\infty}$-algebras the derived sSet-tensoring is weakly equivalent to the underived one. The third step is a direct computation using the explicit formula for the latter from [41]. One concludes that $\mathfrak{A} \simeq \mathbb{L} j ! j^{*} \mathfrak{A}$ are weakly equivalent because the 1-shifted $\mathbb{R}$-valued normalized chains $N_{*}(\operatorname{Sing}(M), \mathbb{R}) \otimes \mathbb{R}[1]$ are weakly equivalent to $\Omega_{c}^{\bullet}(M)[-1]$ as a consequence of de Rham's theorem. Hence, this toymodel also satisfies the homotopy $j$-locality condition from Example 4.12.

Open Problem 4.25. The examples considered above are only toy-models for the kind of homotopy AQFTs that we are eventually interested in. This is because they are 1.) "too topological" in the sense of being weakly equivalent to a constant diagram and 2.) "not quantum" in the sense that they assign only homotopy-coherently commutative observable algebras. It is an open problem to evaluate the derived extension functor $\mathbb{L} j$ ! and test homotopy $j$-locality for more realistic full orthogonal subcategory embeddings, e.g. $j: \overline{\mathbf{L o c}_{\diamond}} \rightarrow \overline{\text { Loc }}$ from Example 2.2, which is crucial for Lorentzian AQFTs.

\subsection{Examples from homotopy invariants}

We present another class of examples of homotopy AQFTs in the sense of Definition 4.18. Let $\overline{\mathbf{C}}$ be an orthogonal category and $\pi: \mathbf{D} \rightarrow \mathbf{C}$ a category fibered in groupoids over its underlying category $\mathbf{C}$. Endowing $\mathbf{D}$ with the pullback orthogonality relation, we obtain an orthogonal functor $\pi: \overline{\mathbf{D}} \rightarrow \overline{\mathbf{C}}$ that we call an orthogonal category fibered in groupoids. The basic idea behind our construction below is as follows: Given any strict $\mathbf{C h}(k)$-valued AQFT $\mathfrak{A} \in \mathbf{Q F T}(\overline{\mathbf{D}})$ on the total category $\overline{\mathbf{D}}$, we would like to construct an AQFT $\mathfrak{A}_{\pi}$ on the base category $\overline{\mathbf{C}}$ by forming homotopy invariants along the groupoid fibers $\pi^{-1}(c) \in \mathbf{G r p d}$, for all $c \in \mathbf{C}$. We shall formalize this construction and show that it naturally leads to a homotopy AQFT $\mathfrak{A}_{\pi} \in \mathbf{Q F T}_{\infty}(\overline{\mathbf{C}})$ on $\overline{\mathbf{C}}$.

Remark 4.26. The physical motivation behind this construction is as follows: As usual, $\overline{\mathbf{C}}$ is interpreted as a category of spacetimes. The total category $\overline{\mathbf{D}}$ of the orthogonal category fibered in groupoids $\pi: \overline{\mathbf{D}} \rightarrow \overline{\mathbf{C}}$ should be interpreted as a category of spacetimes with additional geometric structures (which we call background fields), e.g. spin structures, bundles and connections. The functor $\pi$ forgets this extra structure and hence its fibers $\pi^{-1}(c) \in$ Grpd are the groupoids of background fields on the spacetime $c \in \mathbf{C}$. Note that the morphisms in these groupoids are interpreted as gauge transformations of the background fields, cf. Section 3.1. Our construction assigns to an AQFT $\mathfrak{A} \in \mathbf{Q F T}(\overline{\mathbf{D}})$ on spacetimes with background fields a homotopy AQFT $\mathfrak{A}_{\pi} \in \mathbf{Q F T}_{\infty}(\overline{\mathbf{C}})$ on plain spacetimes. This is achieved by assigning to each $c \in \mathbf{C}$ the homotopy invariants of $\mathfrak{A}$ along the action of the groupoid $\pi^{-1}(c)$ of background fields over $c$. We refer to [10] for concrete examples and more details on the physical interpretation.

Without loss of generality, we can focus on the case where our category fibered in groupoids is given by the Grothendieck construction $\pi: \mathbf{C}_{F} \rightarrow \mathbf{C}$ of a presheaf of groupoids $F: \mathbf{C}^{\text {op }} \rightarrow$ Grpd on $\mathbf{C}$. This follows from the strictification theorems in [48]. Forming homotopy invariants along the groupoid fibers can be described by a homotopy right Kan extension hoRan ${ }_{\pi}: \mathbf{C h}(k) \mathbf{C}_{F} \rightarrow \mathbf{C h}(k)^{\mathbf{C}}$ along $\pi: \mathbf{C}_{F} \rightarrow \mathbf{C}$ of the underlying $\mathbf{C h}(k)$-valued functor of an AQFT $\mathfrak{A} \in \mathbf{Q F T}\left(\overline{\mathbf{C}_{F}}\right)$. By $[10,15]$, we have the following explicit model.

Proposition 4.27. Let $F: \mathbf{C}^{\mathrm{op}} \rightarrow \mathbf{G r p d}$ be a presheaf of groupoids and $\mathfrak{A}: \mathbf{C}_{F} \rightarrow \mathbf{C h}(k)$ a chain complex valued functor on the corresponding Grothendieck construction. Then the homotopy right Kan extension $\mathfrak{A}_{\pi}:=\operatorname{hoRan}_{\pi} \mathfrak{A}:$ $\mathbf{C} \rightarrow \mathbf{C h}(k)$ along the projection functor $\pi: \mathbf{C}_{F} \rightarrow \mathbf{C}$ can be 
computed object-wise by the end

$\mathfrak{A}_{\pi}(c)=\int_{x \in F(c)}\left[N_{*}(B(F(c) \downarrow x), k), \mathfrak{A}(c, x)\right]$,

for all $c \in \mathbf{C}$. Here $N_{*}(-, k)$ denotes the normalized chain functor, [-, - ] the internal hom in $\mathbf{C h}(k)$ and $B(F(c) \downarrow x) \in$ sSet the nerve of the over-category $F(c) \downarrow x$.

Remark 4.28. Note that $\mathfrak{A}_{\pi}(c)$ can also be understood as the homotopy limit

$\mathfrak{A}_{\pi}(c) \simeq \operatorname{holim}\left(\left.\mathfrak{A}\right|_{\pi^{-1}(c)}: F(c) \rightarrow \mathbf{C h}(k)\right)$

of the restriction of $\mathfrak{A}$ to the groupoid fiber $\pi^{-1}(c) \simeq F(c)$. This is important for our interpretation of $\mathfrak{A}_{\pi}=\operatorname{hoRan}_{\pi} \mathfrak{A}$ as forming fiber-wise homotopy invariants.

The main result of this section is that the collection of chain complexes $\mathfrak{A}_{\pi}(c)$, for $c \in \mathbf{C}$, obtained with the construction above carries the structure of a homotopy AQFT in a canonical way. The key ingredient for the proof is the result in [16] that the normalized chain complex $N_{*}(S, k)$ of a simplicial set $S$ carries a canonical $\mathscr{E}_{\infty}$-coaction. This fact, combined with the original $\mathscr{O}_{\overline{\mathbf{C}_{F}}}$-algebra structure on $\mathfrak{A} \in \mathbf{Q F T}\left(\overline{\mathbf{C}_{F}}\right)$, leads to the next theorem. We refer to [15] for a detailed proof.

Theorem 4.29. Let $\overline{\mathbf{C}}$ be an orthogonal category and $F$ : $\mathrm{C}^{\mathrm{op}} \rightarrow \mathbf{G r p d}$ a presheaf of groupoids. Consider the orthogonal category fibered in groupoids $\pi: \overline{\mathbf{C}_{F}} \rightarrow \overline{\mathbf{C}}$ that is obtained by the Grothendieck construction of F. For every strict $\mathbf{C h}(k)$-valued AQFT $\mathfrak{A} \in \mathbf{Q F T}\left(\overline{\mathbf{C}_{F}}\right)$ on the total category, the family of chain complexes $\mathfrak{A}_{\pi}(c) \in \mathbf{C h}(k)$ from Proposition 4.27 carries canonically the structure of an $\mathscr{O}_{\overline{\mathbf{C}}} \otimes \mathscr{E}_{\infty}$-algebra. Hence, $\mathfrak{A}_{\pi} \in \mathbf{Q F T}_{\infty}(\overline{\mathbf{C}})$ is a homotopy AQFT in the sense of Definition 4.18.

Remark 4.30. From a mathematical perspective, the homotopy AQFT $\mathfrak{A}_{\pi} \in \mathbf{Q F T}_{\infty}(\overline{\mathbf{C}})$ from Theorem 4.29 can be interpreted in terms of fiber-wise normalized cochain algebras on $\pi: \overline{\mathbf{C}_{F}} \rightarrow \overline{\mathbf{C}}$ with coefficients in the strict $\mathbf{C h}(k)$ valued AQFT $\mathfrak{A} \in \mathbf{Q F T}\left(\overline{\mathbf{C}_{F}}\right)$. In other words, it is the fiberwise groupoid cohomology of $\pi: \overline{\mathbf{C}_{F}} \rightarrow \overline{\mathbf{C}}$ with coefficients $\mathfrak{A} \in \mathbf{Q F T}\left(\overline{\mathbf{C}_{F}}\right)$. Similarly to ordinary groupoid cohomology, the results of this construction can be interesting even when the coefficients are concentrated in degree 0 . Physical examples of this type have been discussed in [10] and they include e.g. the case of Dirac fields on the groupoid of all possible spin structures over a spacetime.

\section{A On the cosheaf condition in AQFT}

In this appendix we shall analyze an analogue of the cosheaf condition (1.2) for a simple toy-model of an AQFT.
Our main message will be that it is very hard to find covers for which this condition holds true. This motivates and justifies our alternative descent condition that we have sketched at the end of Section 1 and stated precisely in Definition 2.17.

The toy-model we consider is given by the scalar field on the circle $\mathbb{S}^{1}$, which is not a Lorentzian AQFT in the sense of Definition 1.1, but rather a chiral conformal AQFT on the compactified light ray. (In particular, it is an AQFT in the general sense of Definition 2.3.) Denoting by $\operatorname{Open}\left(\mathbb{S}^{1}\right)$ the category of all open subsets of the circle $\mathbb{S}^{1}$, our model is described by a functor $\mathfrak{A}: \operatorname{Open}\left(\mathbb{S}^{1}\right) \rightarrow{ }^{*} \mathbf{A l g}$ to the category of $*$-algebras. To an open subset $U \subseteq \mathbb{S}^{1}$, it assigns the $*$-algebra $\mathfrak{A}(U)$ presented by the following generators and relations:

- Generators: $\Phi_{U}(\varphi)$, for all compactly supported functions $\varphi \in C_{c}^{\infty}(U)$;

- Relations:

- $\mathbb{R}$-linearity: $\Phi_{U}(\alpha \varphi+\beta \psi)=\alpha \Phi_{U}(\varphi)+\beta \Phi_{U}(\psi)$, for all $\alpha, \beta \in \mathbb{R}$ and $\varphi, \psi \in C_{c}^{\infty}(U) ;$

- Hermiticity: $\Phi_{U}(\varphi)^{*}=\Phi_{U}(\varphi)$, for all $\varphi \in C_{c}^{\infty}(U)$;

- CCR: $\left[\Phi_{U}(\varphi), \Phi_{U}(\psi)\right]=\mathrm{i} \int_{\mathbb{S}^{1}} \varphi \mathrm{d} \psi \mathbb{1}$, for all $\varphi, \psi \in$ $C_{c}^{\infty}(U)$.

To an open subset inclusion $\iota_{U}^{V}: U \subseteq V$, the functor assigns the *-homomorphism $\mathfrak{A}\left(\iota_{U}^{V}\right): \mathfrak{A}(U) \rightarrow \mathfrak{A}(V)$ that is defined on the generators by $\Phi_{U}(\varphi) \mapsto \Phi_{V}(\varphi)$. We note that $\mathfrak{A}$ satisfies the following variant of a causality condition (or $\perp$-commutativity condition in the sense of Definition 2.3); For every pair of disjoint open subsets $U_{1}, U_{2} \subseteq V$ of some open subset $V \subseteq \mathbb{S}^{1}$, the induced commutator

$$
\begin{aligned}
{\left[\mathfrak{A}\left(\iota_{U_{1}}^{V}\right)\left(\Phi_{U_{1}}(\varphi)\right), \mathfrak{A}\left(\iota_{U_{2}}^{V}\right)\left(\Phi_{U_{2}}(\psi)\right)\right]_{\mathfrak{A}(V)} } \\
=\left[\Phi_{V}(\varphi), \Phi_{V}(\psi)\right]_{\mathfrak{A}(V)}=\mathrm{i} \int_{\mathbb{S}^{1}} \varphi \mathrm{d} \psi \mathbb{1}=0
\end{aligned}
$$

is zero, for all $\varphi \in C_{c}^{\infty}\left(U_{1}\right)$ and $\psi \in C_{c}^{\infty}\left(U_{2}\right)$, because the integrand is zero as a consequence of $U_{1} \cap U_{2}=\varnothing$.

For any open cover $\left\{U_{i} \subseteq \mathbb{S}^{1}\right\}$ of the circle, we define the $*$-algebra

$\operatorname{colim} \mathfrak{A}\left(U_{\bullet}\right):=\operatorname{colim}\left(\coprod_{i j} \mathfrak{A}\left(U_{i j}\right) \rightrightarrows \coprod_{i} \mathfrak{A}\left(U_{i}\right)\right)$,

which also admits a simple presentation by generators and relations:

- Generators: $\Phi_{U_{i}}(\varphi)$, for all $i$ and all $\varphi \in C_{c}^{\infty}\left(U_{i}\right)$;

- Relations:

- $\mathbb{R}$-linearity: $\Phi_{U_{i}}(\alpha \varphi+\beta \psi)=\alpha \Phi_{U_{i}}(\varphi)+\beta \Phi_{U_{i}}(\psi)$, for all $i$, all $\alpha, \beta \in \mathbb{R}$ and all $\varphi, \psi \in C_{c}^{\infty}\left(U_{i}\right)$;

- Hermiticity: $\Phi_{U_{i}}(\varphi)^{*}=\Phi_{U_{i}}(\varphi)$, for all $i$ and all $\varphi \in$ $C_{c}^{\infty}\left(U_{i}\right)$ 
- CCR: $\left[\Phi_{U_{i}}(\varphi), \Phi_{U_{i}}(\psi)\right]=\mathrm{i} \int_{\mathbb{S}^{1}} \varphi \mathrm{d} \psi \mathbb{1}$, for all $i$ and all $\varphi, \psi \in C_{c}^{\infty}\left(U_{i}\right)$

- Overlap relations: $\Phi_{U_{i}}(\varphi)=\Phi_{U_{j}}(\varphi)$, for all $i, j$ and all $\varphi \in C_{c}^{\infty}\left(U_{i j}\right)$.

Note that there are no a priori commutation relations between $\Phi_{U_{i}}(\varphi)$ and $\Phi_{U_{j}}(\psi)$ for different $i \neq j$. Depending on the cover, there however exist certain induced commutation relations that result by combining the CCRs for individual $i$ 's and the overlap relations.

There exists a canonical $*$-homomorphism

$\operatorname{colim} \mathfrak{A}\left(U_{\bullet}\right) \longrightarrow \mathfrak{A}\left(\mathbb{S}^{1}\right), \Phi_{U_{i}}(\varphi) \longmapsto \Phi_{\mathbb{S}^{1}}(\varphi)$

to the $*$-algebra on the full circle. We would like to answer the question for which covers $\left\{U_{i} \subseteq \mathbb{S}^{1}\right\}$ this is an isomorphism, i.e. for which covers the cosheaf condition holds true for our example.

Proposition A.1. (A.3) is an isomorphism if and only if the open cover $\left\{U_{i} \subseteq \mathbb{S}^{1}\right\}$ satisfies the condition

$\forall i, j \exists k: U_{i} \cup U_{j} \subseteq U_{k}$

Proof. Choosing a partition of unity $\sum_{i} \chi_{i}=1$ subordinate to $\left\{U_{i} \subseteq \mathbb{S}^{1}\right\}$, we define for each $\varphi \in C_{c}^{\infty}\left(\mathbb{S}^{1}\right)$ an element

$\widetilde{\Phi}(\varphi):=\sum_{i} \Phi_{U_{i}}\left(\chi_{i} \varphi\right) \in \operatorname{colim} \mathfrak{A}\left(U_{\bullet}\right)$

These elements are independent of the choice of partition of unity: For any other choice $\sum_{i} \rho_{i}=1$, we obtain

$$
\begin{aligned}
\sum_{i} \Phi_{U_{i}}\left(\rho_{i} \varphi\right) & =\sum_{i, j} \Phi_{U_{i}}\left(\rho_{i} \chi_{j} \varphi\right)=\sum_{i, j} \Phi_{U_{j}}\left(\rho_{i} \chi_{j} \varphi\right) \\
& =\sum_{j} \Phi_{U_{j}}\left(\chi_{j} \varphi\right)=\widetilde{\Phi}(\varphi),
\end{aligned}
$$

where in the second step we used the overlap relations for $\rho_{i} \chi_{j} \varphi \in C_{c}^{\infty}\left(U_{i j}\right)$. The elements in (A.5) are clearly $\mathbb{R}$ linear in $\varphi \in C_{c}^{\infty}\left(\mathbb{S}^{1}\right)$ and Hermitian. Moreover, we have that $\widetilde{\Phi}(\varphi) \mapsto \Phi_{\mathbb{S}^{1}}(\varphi)$ under the map (A.3). It follows that (A.3) is an isomorphism, with inverse $\Phi_{\mathbb{S}^{1}}(\varphi) \mapsto \widetilde{\Phi}(\varphi)$, if and only if the $\widetilde{\Phi}(\varphi)$ 's, for $\varphi \in C_{c}^{\infty}\left(\mathbb{S}^{1}\right)$, satisfy the CCR. These are equivalent to the commutation relations

$$
\left[\Phi_{U_{i}}(\varphi), \Phi_{U_{j}}(\psi)\right]=\mathrm{i} \int_{\mathbb{S}^{1}} \varphi \mathrm{d} \psi \mathbb{1}
$$

in $\operatorname{colim} \mathfrak{A}\left(U_{\bullet}\right)$, for all $i, j$ and all $\varphi \in C_{c}^{\infty}\left(U_{i}\right)$ and $\psi \in$ $C_{c}^{\infty}\left(U_{j}\right)$, which are satisfied if and only if condition (A.4) holds.

Lemma A.2. An open cover $\left\{U_{i} \subseteq \mathbb{S}^{1}\right\}$ satisfies (A.4) if and only if one of its members is the whole circle $\mathbb{S}^{1}$.
Proof. The direction " $\Leftarrow$ " is obvious. To prove " $\Rightarrow$ ", note that each open cover of the compact space $\mathbb{S}^{1}$ has a finite subcover, say $U_{1}, \ldots, U_{N} \subseteq \mathbb{S}^{1}$. Applying (A.4) iteratively, we obtain $\mathbb{S}^{1}=U_{1} \cup U_{2} \cup \cdots \cup U_{N} \subseteq U_{i} \cup U_{3} \cup \cdots \cup U_{N} \subseteq \cdots \subseteq$ $U_{k}$, i.e. there exists $k$ such that $U_{k}=\mathbb{S}^{1}$ is the circle.

Corollary A.3. The AQFT $\mathfrak{A}:$ Open $\left(\mathbb{S}^{1}\right) \rightarrow{ }^{*}$ Alg describing the scalar field on $\mathbb{S}^{1}$ satisfies the cosheaf condition for an open cover $\left\{U_{i} \subseteq \mathbb{S}^{1}\right\}$ if and only if $\mathbb{S}^{1}$ is a member of this cover. In particular, it does not satisfy the cosheaf condition for any open cover $\left\{I_{i} \subseteq \mathbb{S}^{1}\right\}$ by intervals.

Remark A.4. Clearly, a cosheaf condition for covers containing the entire space holds trivially and hence has no power. From the discussion above we deduce that even for an elementary prototypical example of AQFT, such as the scalar field on the circle $\mathbb{S}^{1}$, the cosheaf condition holds only for covers that contain the circle itself. A similar behavior arises more generally also in AQFTs on Lorentzian manifolds. The alternative descent condition $j$-locality, inspired by Fredenhagen's universal algebra construction [36-38] and formalized in Definition 2.17, is better behaved in standard examples of AQFTs, cf. Example 2.20.

Acknowledgements. We would like to thank our collaborators S. Bruinsma, M. Perin, U. Schreiber, R. Szabo and L. Woike for their work and contributions to the homotopical algebraic quantum field theory program that we initiated some years ago. A.S. also would like to thank the organizers and sponsors of the LMS-EPSRC Durham Symposium Higher Structures in $M$-Theory for this very interesting and fruitful conference. The work of M.B. is supported by a research grant funded by the Deutsche Forschungsgemeinschaft (DFG, Germany). A.S. gratefully acknowledges the financial support of the Royal Society (UK) through a Royal Society University Research Fellowship, a Research Grant and an Enhancement Award.

Key words. Algebraic quantum field theory, gauge theory, model categories, operads, stacks.

\section{References}

[1] M. Atiyah, "Topological quantum field theories," Inst. Hautes Etudes Sci. Publ. Math. 68, 175 (1989).

[2] D. Ayala and J. Francis, "Factorization homology of topological manifolds," J. Topol. 8, no. 4, 1045-1084 (2015) [arXiv:1206.5522 [math.AT]].

[3] C. Bär, N. Ginoux and F. Pfäffle, Wave equations on Lorentzian manifolds and quantization, Eur. Math. Soc., Zürich (2007) [arXiv:0806.1036 [math.DG]].

[4] C. Becker, M. Benini, A. Schenkel and R. J. Szabo, "Abelian duality on globally hyperbolic space- 
times," Commun. Math. Phys. 349, no. 1, 361 (2017) [arXiv:1511.00316 [hep-th]].

[5] C. Becker, A. Schenkel and R. J. Szabo, "Differential cohomology and locally covariant quantum field theory,” Rev. Math. Phys. 29, no. 01, 1750003 (2016) [arXiv:1406.1514 [hep-th]].

[6] M. Benini, C. Dappiaggi, T. P. Hack and A. Schenkel, "A $C^{*}$-algebra for quantized principal $U(1)$ connections on globally hyperbolic Lorentzian manifolds," Commun. Math. Phys. 332, 477 (2014) [arXiv:1307.3052 [math-ph]].

[7] M. Benini, C. Dappiaggi and A. Schenkel, "Quantized Abelian principal connections on Lorentzian manifolds," Commun. Math. Phys. 330, 123 (2014) [arXiv:1303.2515 [math-ph]].

[8] M. Benini, C. Dappiaggi and A. Schenkel, "Algebraic quantum field theory on spacetimes with timelike boundary," Annales Henri Poincaré 19, no. 8, 2401 (2018) [arXiv:1712.06686 [math-ph]].

[9] M. Benini and A. Schenkel, "Poisson algebras for non-linear field theories in the Cahiers topos," Annales Henri Poincaré 18, no. 4, 1435 (2017) [arXiv:1602.00708 [math-ph]].

[10] M. Benini and A. Schenkel, "Quantum field theories on categories fibered in groupoids," Commun. Math. Phys. 356, no. 1, 19 (2017) [arXiv:1610.06071 [math$\mathrm{ph}]$.

[11] M. Benini, A. Schenkel and U. Schreiber, “The Stack of Yang-Mills Fields on Lorentzian Manifolds," Commun. Math. Phys. 359, no. 2, 765 (2018) [arXiv:1704.01378 [math-ph]].

[12] M. Benini, A. Schenkel and R. J. Szabo, "Homotopy colimits and global observables in Abelian gauge theory," Lett. Math. Phys. 105, no. 9, 1193 (2015) [arXiv:1503.08839 [math-ph]].

[13] M. Benini, A. Schenkel and L. Woike, "Operads for algebraic quantum field theory,” arXiv:1709.08657 [math-ph].

[14] M. Benini, A. Schenkel and L. Woike, "Involutive categories, colored $*$-operads and quantum field theory," arXiv:1802.09555 [math.CT].

[15] M. Benini, A. Schenkel and L. Woike, "Homotopy theory of algebraic quantum field theories," arXiv:1805.08795 [math-ph].

[16] C. Berger and B. Fresse, "Combinatorial operad actions on cochains,” Math. Proc. Cambridge Philos. Soc. 137, no. 1, 135-174 (2004) [arXiv:math/0109158 [math.AT]].

[17] C. Berger and I. Moerdijk, "Axiomatic homotopy theory for operads," Comment. Math. Helv. 78, 805831 (2003) [arXiv:math/0206094 [math.AT]].

[18] C. Berger and I. Moerdijk, "Resolution of coloured operads and rectification of homotopy algebras," in: A. Davydov, M. Batanin, M. Johnson, S. Lack and A. Neeman (eds.), Categories in algebra, geometry and mathematical physics, Contemp. Math. 431, 3158, American Mathematical Society, Providence, RI (2007).
[19] S. Bruinsma, "Coloring operads for algebraic field theory," submitted.

[20] S. Bruinsma and A. Schenkel, "Algebraic field theory operads and linear quantization,” arXiv:1809.05319 [math-ph].

[21] R. Brunetti, C. Dappiaggi, K. Fredenhagen and J. Yngvason, Advances in algebraic quantum field theory, Springer Verlag, Heidelberg (2015).

[22] R. Brunetti, M. Duetsch and K. Fredenhagen, "Perturbative Algebraic Quantum Field Theory and the Renormalization Groups," Adv. Theor. Math. Phys. 13, no. 5, 1541 (2009) [arXiv:0901.2038 [math-ph]].

[23] R. Brunetti, K. Fredenhagen and R. Verch, "The generally covariant locality principle: A new paradigm for local quantum field theory," Commun. Math. Phys. 237, 31 (2003) [math-ph/0112041].

[24] D. Calaque, T. Pantev, B. Toën, M. Vaquié and G. Vezzosi, "Shifted Poisson structures and deformation quantization,” J. Topol. 10, no. 2, 483-584 (2017) [arXiv:1506.03699 [math.AG]].

[25] D.-C. Cisinski and I. Moerdijk, "Dendroidal sets and simplicial operads,” J. Topol. 6, no. 3, 705-756 (2013) [arXiv:1109.1004 [math.AT]].

[26] K. Costello and O. Gwilliam, Factorization algebras in quantum field theory. Vol. 1, New Mathematical Monographs 31, Cambridge University Press, Cambridge (2017).

[27] M. Crainic, "Differentiable and algebroid cohomology, van Est isomorphisms, and characteristic classes," Comment. Math. Helv. 78, no. 4, 681-721 (2003).

[28] C. Dappiaggi and B. Lang, "Quantization of Maxwell's equations on curved backgrounds and general local covariance,” Lett. Math. Phys. 101, 265 (2012) [arXiv:1104.1374 [gr-qc]].

[29] J. Dougherty, "Sameness and Separability in Gauge Theories,” Philosophy of Science 84, no. 5, 1189-1201 (2017).

[30] D. Dugger, "Universal homotopy theories," Adv. Math. 164, no. 1, 144-176 (2001) [arXiv:math/0007070 [math.AT]].

[31] D. Dugger, S. Hollander and D. C. Isaksen, "Hypercovers and simplicial presheaves," Math. Proc. Cambridge Philos. Soc. 136, no. 1, 9-51 (2004) [arXiv:math/0205027 [math.AT]].

[32] W. G. Dwyer and J. Spalinski, "Homotopy theories and model categories,” in: I. M. James (ed.), Handbook of Algebraic Topology, 73, North-Holland, Amsterdam (1995).

[33] J. M. Egger, "On involutive monoidal categories," Theory and Applications of Categories 25, no. 14, 368 (2011).

[34] J. M. Egger, “On the definition of $C^{*}$-algebra," available at http:// cheng.staff.shef.ac.uk/pssl85/egger.pdf

[35] D. Fiorenza, H. Sati and U. Schreiber, "A higher stacky perspective on Chern-Simons theory," in: D. Calaque and T. Strobl (eds.), Mathematical Aspects of Quantum Field Theories, Mathematical Physics Studies, 
Springer (2015) [arXiv:1301.2580 [hep-th]].

[36] K. Fredenhagen, "Generalizations of the theory of superselection sectors," in: D. Kastler (ed.), The algebraic theory of superselection sectors: Introduction and recent results, World Scientific Publishing, 379 (1990).

[37] K. Fredenhagen, "Global observables in local quantum physics,” in: H. Araki, K. R. Ito, A. Kishimoto and I. Ojima (eds.), Quantum and non-commutative analysis: Past, present and future perspectives, 41-51, Kluwer Academic Publishers (1993).

[38] K. Fredenhagen, K.-H. Rehren and B. Schroer, "Superselection sectors with braid group statistics and exchange algebras II: Geometric aspects and conformal covariance," Rev. Math. Phys. 4, 113 (1992).

[39] K. Fredenhagen and K. Rejzner, "Batalin-Vilkovisky formalism in the functional approach to classical field theory," Commun. Math. Phys. 314, 93 (2012) [arXiv:1101.5112 [math-ph]].

[40] K. Fredenhagen and K. Rejzner, "Batalin-Vilkovisky formalism in perturbative algebraic quantum field theory," Commun. Math. Phys. 317, 697 (2013) [arXiv:1110.5232 [math-ph]].

[41] B. Fresse, "The cotriple resolution of differential graded algebras,” Proc. Amer. Math. Soc. 144, 46934707 (2016) [arXiv:1503.08489 [math.AT]]

[42] B. Fresse, Homotopy of operads and GrothendieckTeichmüller groups. Part 1: The algebraic theory and its topological background, Mathematical Surveys and Monographs 217, American Mathematical Society, Providence, RI (2017).

[43] G. Ginot, T. Tradler and M. Zeinalian, "Derived Higher Hochschild Homology, Topological Chiral Homology and Factorization Algebras," Commun. Math. Phys. 326, 635-686 (2014) [arXiv:1011.6483 [math.QA]].

[44] R. Haag and D. Kastler, "An algebraic approach to quantum field theory,” J. Math. Phys. 5, 848 (1964).

[45] V. Hinich, "Homological algebra of homotopy algebras," Comm. Algebra 25, no. 10, 3291-3323 (1997) [arXiv:q-alg/9702015]. Erratum: arXiv:math/0309453 [math.QA].

[46] V. Hinich, "Rectification of algebras and modules," Doc. Math. 20, 879-926 (2015) [arXiv:1311.4130 [math.QA]].

[47] P. S. Hirschhorn, Model categories and their localizations, Math. Surveys Monogr. 99, Amer. Math. Soc., Providence, RI (2003).

[48] S. Hollander, "A homotopy theory for stacks," Israel J. Math. 163, 93-124 (2008) [arXiv:math.AT/0110247].

[49] S. Hollands, "Renormalized Quantum Yang-Mills Fields in Curved Spacetime,” Rev. Math. Phys. 20, 1033 (2008) [arXiv:0705.3340 [gr-qc]].

[50] M. Hovey, Model categories, Math. Surveys Monogr. 63, Amer. Math. Soc., Providence, RI (1999).

[51] M. Kashiwara and P. Schapira, Categories and sheaves, Springer Verlag, Berlin (2006).

[52] Y. Kawahigashi, "Conformal Field Theory, Tensor
Categories and Operator Algebras," J. Phys. A 48, no. 30, 303001 (2015) [arXiv:1503.05675 [math-ph]].

[53] B. Lang, Universal constructions in algebraic and locally covariant quantum field theory, $\mathrm{PhD}$ thesis, University of York (2014).

[54] J. Lurie, "On the classification of topological field theories," Current Developments in Mathematics, Volume 2008, 129-280 (2009) [arXiv:0905.0465 [math.CT]].

[55] J. Lurie, Higher topos theory, Annals of Mathematics Studies 170, Princeton University Press (2009).

[56] J. Lurie, Higher algebra, book draft available at http: //www.math.harvard.edu/ lurie/papers/HA.pdf

[57] J. Nguyen, N. J. Teh and L. Wells, "Why surplus structure is not superfluous," to appear in The British Journal for the Philosophy of Science [arXiv:1712.01228 [physics.hist-ph]].

[58] T. Pantev, B. Toën, M. Vaquié and G. Vezzosi, "Shifted symplectic structures,” Publ. Math. Inst. Hautes Études Sci. 117, 271-328 (2013) [arXiv:1111.3209 [math.AG]].

[59] D. Pavlov and J. Scholbach, "Admissibility and rectification of colored symmetric operads," Journal of Topology 11, 559-601 (2018) [arXiv:1410.5675 [math.AT]].

[60] K. Rejzner, Perturbative algebraic quantum field theory: An introduction for mathematicians, Mathematical Physics Studies, Springer, Cham (2016).

[61] U. Schreiber, "Differential cohomology in a cohesive infinity-topos," current version available at https://ncatlab.org/schreiber/show/differential+ cohomology+in+a+cohesive+topos [arXiv:1310.7930 [math-ph]].

[62] C. Schweigert and L. Woike, "Extended Homotopy Quantum Field Theories and their Orbifoldization," arXiv:1802.08512 [math.QA].

[63] M. Spitzweck, Operads, algebras and modules in general model categories, PhD Thesis, Bonn (2001). Available at http://hss.ulb.uni-bonn.de/2001/0241/ 0241.pdf

[64] M. T. Tehrani and J. Zahn, "Background independence in gauge theories," arXiv:1804.07640 [mathph].

[65] D. Yau, Colored operads, Graduate Studies in Mathematics 170, American Mathematical Society, Providence, RI (2016).

[66] D. Yau, "Homotopical Quantum Field Theory," arXiv:1802.08101 [math-ph]. 